\title{
VERTEX ALGEBRAS AND COORDINATE RINGS OF SEMI-INFINITE FLAGS
}

\author{
EVGENY FEIGIN AND IEVGEN MAKEDONSKYI
}

\begin{abstract}
The direct sum of irreducible level one integrable representations of affine Kac-Moody Lie algebra of (affine) type $A D E$ carries a structure of $P / Q$-graded vertex operator algebra. There exists a filtration on this direct sum studied by Kato and Loktev such that the corresponding graded vector space is a direct sum of global Weyl modules. The associated graded space with respect to the dual filtration is isomorphic to the homogenous coordinate ring of semi-infinite flag variety. We describe the ring structure in terms of vertex operators and endow the homogenous coordinate ring with a structure of $P / Q$-graded vertex operator algebra. We use the vertex algebra approach to derive semi-infinite Plücker-type relations in the homogeneous coordinate ring.
\end{abstract}

\section{INTRODUCTION}

Let $\mathfrak{g}$ be a simple complex Lie algebra of type $A D E$ (i.e. simply-laced). Let $P$ and $Q$ be the weight and root lattices of $\mathfrak{g}$. We denote by $\widehat{\mathfrak{g}}$ the corresponding affine Kac-Moody Lie algebra, which is the central extension of the loop algebra $\mathfrak{g} \otimes \mathbb{C}\left[t, t^{-1}\right]$. It is well known (see e.g. [C, Kac1, Kum]) that the level one integrable irreducible $\widehat{\mathfrak{g}}$-modules are in one-to-one correspondence with the quotient $P / Q$. In particular, the neutral element in $P / Q$ corresponds to the basic (vacuum) representation $L\left(\Lambda_{0}\right)$. We note that if $\mathfrak{g}=\mathfrak{s l}_{n}$, then there are $n$ level one integrable $\widehat{\mathfrak{g}}$-modules: $L\left(\Lambda_{0}\right)$ and one module for each fundamental weight of $\mathfrak{g}$. And if $\mathfrak{g}$ is of type $E_{8}$, then $P=Q$ and the only integrable level one module is the basic one.

The basic representation $L\left(\Lambda_{0}\right)$ of $\widehat{\mathfrak{g}}$ carries a structure of vertex operator algebra (VOA) $\mathrm{FK}, \mathrm{FB}, \mathrm{Kac2}$. In short, a VOA is a correspondence between vectors in $L\left(\Lambda_{0}\right)$ and fields on the same space (the formal Taylor series with coefficients in $\left.\operatorname{End}\left(L\left(\Lambda_{0}\right)\right)\right)$. In particular, the action of the generators of $\widehat{\mathfrak{g}}$ is expressed in terms of the components of certain fields. For our purposes we need a generalization of the standard definition of vertex operator algebra, the so called $\Gamma$-graded VOA for a finite group $\Gamma$ (this notion generalizes the notion of super vertex algebra) (see $[\mathrm{DL}, \mathrm{R}, \overline{\mathrm{BK}},[\mathrm{S}]$ ). We note that we only use the case $\Gamma=P / Q$. The space of states $\mathbb{L}$ of the $P / Q$ graded VOA we are working with is equal to the direct sum of all irreducible level one integrable $\widehat{\mathfrak{g}}$-modules (in particular, $\mathbb{L}$ is naturally graded by the group $P(Q)$. To an element $A \in \mathbb{L}$ one attaches a field $Y(A, z)$ acting on $\mathbb{L}$. An important difference between the $\Gamma$-graded VOAs and their classical 
analogues is that the fields $Y(A, z)$ contain rational (not necessarily integer) powers of $z$. We use these fields in order to study certain structure on level one modules which involves Weyl modules: a class of representations of the current algebra $\mathfrak{g} \otimes \mathbb{C}[t]$.

The current algebra $\mathfrak{g} \otimes \mathbb{C}[t]$ can be seen as an intermediate step between $\mathfrak{g}$ and $\widehat{\mathfrak{g}}$. In particular, its representation theory (algebraic, geometric and combinatorial) has various deep connections with representation theory of $\mathfrak{g}$ and that of $\widehat{\mathfrak{g}}$ (see e.g. [CL, FL, FM1, KL]). The current algebra has two important classes of cyclic representations: local Weyl modules $W_{\lambda}$ and global Weyl modules $\mathbb{W}_{\lambda}([\mathrm{CP},[\mathrm{CFK}, \overline{F M O}])$. Both classes are labeled by the same parameter $\lambda \in P_{+}$(the dominant cone of the weight lattice), but the local modules are finite-dimensional, while the global Weyl modules are infinite-dimensional. These modules have many interesting features, in particular, they are important for the study of nonsymmetric Macdonald polynomials Ch, CI, I, FKM, Kat, ChK and semi-infinite flag varieties [BF1, BF2, FMi, KNS].

In a recent paper $\mathrm{KL}$ the authors studied a filtration $F_{\lambda} \subset \mathbb{L}$ labeled by dominant weights $\lambda \in P_{+}$, where each $F_{\lambda}$ is a thick Demazure module (a subspace generated from an extremal weight $\lambda$ vector of $\mathbb{L}$ by the action of $\left.\mathfrak{g} \otimes \mathbb{C}\left[t^{-1}\right]\right)$. The main result of [KL for level one representations claims that the associated graded $\operatorname{gr} F_{\bullet}$ is isomorphic to the direct sum of global Weyl modules $\bigoplus_{\lambda \in P_{+}} \mathbb{W}_{\lambda}$ (see also $[\mathrm{ChF}]$ ). We consider the dual filtration $F_{\lambda}^{\dagger}$ on $\mathbb{L}$ (with respect to the Shapovalov form) with the property that $\operatorname{gr} F_{\bullet}^{\dagger}$ is isomorphic to the space $\mathbb{W}^{*}=\bigoplus_{\lambda \in P_{+}} \mathbb{W}_{\lambda}^{*}$.

The advantage of the dual picture is that the space $\mathbb{W}^{*}$ carries an important structure of commutative associative algebra coming from the $\mathfrak{g} \otimes \mathbb{C}[t]$ equivariant surjections $\mathbb{W}_{\lambda}^{*} \otimes \mathbb{W}_{\mu}^{*} \rightarrow \mathbb{W}_{\lambda+\mu}^{*}$ [Kat, $\left[\mathrm{KNS}\right.$. The algebra $\mathbb{W}^{*}$ is the homogeneous coordinate ring of the semi-infinite flag variety of the group $G[[t]]$ with respect to the Drinfeld-Plücker embedding (see [FMi, BF1, BF2]). Our goal is to use the $P / Q$-graded vertex operators structure on $\mathbb{L}$ in order to study the algebra $\mathbb{W}^{*}$.

Let $Y(A, z)=\sum_{n \in \mathbb{Q}} A_{(n)} z^{-n-1}$ be the Fourier expansion of a vertex operator. We first prove the following claim:

$$
A_{(n)} B \in F_{\lambda+\mu}^{\dagger} \text { for all } A \in F_{\lambda}^{\dagger}, B \in F_{\mu}^{\dagger}, n \in \mathbb{Q} \text {. }
$$

We conclude that the algebra $\mathbb{W}^{*}$ carries structure of $P / Q$-graded vertex operator algebra.

The next goal is to describe the filtration $F_{\lambda}^{\dagger}$ in terms of the action of vertex operators. In order to state the result, we consider the natural embeddings $W_{\omega_{i}}^{*} \subset \mathbb{W}_{\omega_{i}}^{*}$ for all fundamental weights $\omega_{i}$. Using $\operatorname{gr} F_{\lambda}^{\dagger} \simeq \mathbb{W}_{\lambda}^{*}$, we fix a lift of the fundamental local Weyl modules into $F_{\omega_{i}}^{\dagger}$ and consider $W_{\omega_{i}}^{*}$ as sitting inside $F_{\omega_{i}}^{\dagger} \subset \mathbb{L}$. Let $\lambda=\omega_{j_{1}}+\cdots+\omega_{j_{s}}$. We prove that

$$
F_{\lambda}^{\dagger}=\operatorname{span}\left\{A_{\left(n_{1}\right)}^{1} \ldots A_{\left(n_{s}\right)}^{s}|0\rangle, A^{i} \in W_{\omega_{j_{i}}}^{*}\right\}
$$


where $|0\rangle \in L\left(\Lambda_{0}\right) \subset \mathbb{L}$ is the vacuum vector (see [A, Li1, Li2] for similar constructions). As a consequence we endow $\mathbb{W}^{*}$ with the structure of $P / Q$ graded VOA.

Our next task is to describe the multiplication in the algebra $\mathbb{W}^{*}$ in terms of the vertex operators. In short, we write down an explicit formula for the multiplication of the elements from $\mathbb{W}_{\omega_{i}}^{*}$ in terms of the products of vertex operators (see (4.2) for the explicit formula). We apply the resulting formula to derive a set of quadratic relations in the algebra $\mathbb{W}^{*}$. We show that in type $A$ these relations are defining and coincide with those given in [FM2].

The paper is organized as follows. In Section 1 we collect main notation and introduce main objects from the theory of simple, current and affine Lie algebras. We also briefly recall the formalism of semi-infinite flag varieties and Weyl modules. In Section 2 we collect main definitions from the theory of $\Gamma$-graded vertex operator algebras. Section 3 is devoted to the brief description of our constructions and to the strategy of the proofs. In Section 4 we construct vertex operator algebra filtration $(P / Q$-graded analog of the standard filtration) and write down the multiplication formula in terms of vertex operators. Section 5 is devoted to the derivation of the semiinfinite Plücker-type relations in the homogeneous coordinate ring of the semi-infinite flag variety. In Section 6 we show that in type $A$ the relations derived in the previous section are defining.

\section{Semi-Infinite flag varieties, Weyl modules and integrable REPRESENTATIONS}

In this section we collect main definitions and constructions needed in the main body of the paper. The three main ingredients are the PlückerDrinfeld realization of the semi-infinite flag varieties, Weyl modules and the connection between the global Weyl modules and integrable level one representations of the affine Kac-Moody Lie algebra described in KL].

1.1. Simple Lie algebras. Let $\mathfrak{g}$ be a simple Lie algebra of rank $r$ with the Cartan decomposition $\mathfrak{g}=\mathfrak{b} \oplus \mathfrak{n}_{-}$, where $\mathfrak{b}=\mathfrak{n} \oplus \mathfrak{h}$ is a Borel subalgebra. Let $\omega_{1}, \ldots, \omega_{r}$ and $\alpha_{1}, \ldots, \alpha_{r}$ be fundamental weights and simple roots. We denote by $P$ and $P_{+}$the weight lattice and its dominant cone. In particular, $P_{+}=\bigoplus_{i=1}^{r} \mathbb{Z}_{\geq 0} \omega_{i}$. Let $Q$ be the root lattice spanned by $\alpha_{i}, i=1, \ldots, r$. We denote by $\Delta=\Delta_{+} \sqcup \Delta_{-}$the set of roots of $\mathfrak{g}$. For $\alpha \in \Delta_{+}$we denote by $f_{\alpha} \in \mathfrak{n}_{-}, e_{\alpha} \in \mathfrak{n}$ the corresponding Chevalley generators. In what follows we use the following partial order on the lattice $P$ :

$$
\mu \leq \lambda \text { iff } \lambda-\mu=\sum_{i=1}^{r} a_{i} \alpha_{i}, a_{i} \in \mathbb{Q}_{\geq 0} .
$$

We note that $\lambda-\omega_{i}<\lambda$ for any $\lambda \in P, i=1, \ldots, r$. In particular, 0 is the smallest element in $P_{+}$.

For a dominant integral weight $\lambda \in P_{+}$we denote by $V_{\lambda}$ irreducible $\mathfrak{g}$ module of highest weight $\lambda$. In particular, $V_{\lambda}$ contains a highest weight 
vector $v_{\lambda}$ such that $\mathfrak{n} v_{\lambda}=0$. Let $(\cdot, \cdot)$ be the Killing form. For a root $\alpha$ we denote the corresponding coroot by $\alpha^{\vee}$. One has the following (defining) relations in $V_{\lambda}: f_{\alpha}^{\left(\lambda, \alpha^{\vee}\right)+1} v_{\lambda}=0$.

For a weight $\lambda \in P_{+}$the dual representation $V_{\lambda}^{*}$ is isomorphic to a highest weight module. We denote the highest weight of this module by $\lambda^{*}$. One has the equality $\lambda^{*}=-w_{0} \lambda$, where $w_{0}$ is the longest element of the Weyl group of $\mathfrak{g}$. In particular, for any $i=1, \ldots, r$ the weight $\omega_{i}^{*}$ is again a fundamental weight.

Let $G \supset B$ be the Lie groups of $\mathfrak{g}$ and $\mathfrak{b}$. Then the flag variety $G / B$ enjoys the Plücker embedding $G / B \subset \prod_{i=1}^{r} \mathbb{P}\left(V_{\omega_{i}}\right)$. The Plücker embedding gives rise to the family of line bundles $\mathcal{O}(\lambda), \lambda \in P_{+}$on $G / B$ (the tensor products of pull backs of the line bundles $\mathcal{O}(1)$ on $\left.\mathbb{P}\left(V_{\omega_{i}}\right)\right)$. The Borel-Weil theorem states that the space of sections $H^{0}(G / B, \mathcal{O}(\lambda))$ is isomorphic to $V_{\lambda}^{*}$. The coordinate ring of $G / B$ (with respect to the Plücker embedding) is given by $\bigoplus_{\lambda \in P_{+}} V_{\lambda}^{*}$.

1.2. Weyl modules. We consider the current algebra $\mathfrak{g} \otimes \mathbb{C}[t]$. For $x \in \mathfrak{g}$, $k \geq 0$ we denote by $x t^{k}$ the element $x \otimes t^{k} \in \mathfrak{g} \otimes \mathbb{C}[t]$. For a dominant integral weight $\lambda$ let $W_{\lambda}$ be the corresponding local Weyl module of highest weight $\lambda$ and let $\mathbb{W}_{\lambda}$ be the global Weyl module. The global Weyl module $\mathbb{W}_{\lambda}$ is cyclic $\mathfrak{g} \otimes \mathbb{C}[t]$ module with cyclic vector $w_{\lambda}$ of $\mathfrak{h} \otimes 1$ weight $\lambda$ and defining relations

$$
\mathfrak{n} \otimes \mathbb{C}[t] . w_{\lambda}=0,\left(f_{\lambda} \otimes 1\right)^{\left(\lambda, \alpha^{\vee}\right)+1} w_{\lambda}=0 .
$$

The defining relations for the local Weyl module $W_{\lambda}$ differ by the additional relation $\mathfrak{h} \otimes t \mathbb{C}[t] . w_{\lambda}=0$. We note that both local and global Weyl modules are graded, where the grading is defined by saying that the degree of $w_{\lambda}$ is zero and $x \otimes t^{i}$ increases the degree by $i$. We refer to this grading as $q$-grading. For a graded $\mathfrak{g}$-module $M=\bigoplus_{k \in \mathbb{Z}} M[k]$ with finite-dimensional graded components we denote $\operatorname{ch}_{q} M=\sum_{k \in \mathbb{Z}} q^{k} \operatorname{ch} M[k]$. Let $(q)_{n}=\prod_{i=1}^{n}\left(1-q^{i}\right)$.

We have the following properties of Weyl modules.

- $W_{\lambda}$ is finite-dimensional and $\operatorname{dim} W_{\lambda}=\prod_{i=1}^{r}\left(\operatorname{dim} W_{\omega_{i}}\right)^{\left(\lambda, \alpha_{i}^{\vee}\right)}$;

- $\mathrm{U}(\mathfrak{g}) w_{\lambda} \simeq V_{\lambda} \subset W_{\lambda}$;

- $\operatorname{ch} \mathbb{W}_{\lambda}=\operatorname{ch} W_{\lambda} \prod_{i=1}^{r}(q)_{\left(\lambda, \alpha_{i}^{\vee}\right)}^{-1}$.

Remark 1.1. In type $A$ one has $W_{\omega_{i}} \simeq V_{\omega_{i}}$. However, in general $W_{\omega_{i}}$ is not irreducible as $\mathfrak{g}$ module.

It was shown in $\mathrm{Kat}$ that one has $\mathfrak{g} \otimes \mathbb{C}[t]$ equivariant embeddings $\mathbb{W}_{\lambda+\mu} \subset \mathbb{W}_{\lambda} \otimes \mathbb{W}_{\mu}, w_{\lambda+\mu} \mapsto w_{\lambda} \otimes w_{\mu}$. Thus one gets the natural algebra structure on the space $\bigoplus_{\lambda \in P_{+}} \mathbb{W}_{\lambda}^{*}$.

1.3. Construction of global Weyl modules. In this subsection we describe an explicit construction of global Weyl modules and dual global Weyl modules. Let $U$ be a finite-dimensional $\mathfrak{g}[t]$-module (here and below we use 
the shorthand notation $\mathfrak{g}[t]=\mathfrak{g} \otimes \mathbb{C}[t])$. We construct two $\mathfrak{g}[t]$-modules $U[t]$ and $U\left[t^{-1}\right]$ satisfying the following properties:

$$
\operatorname{ch} U[t]=\frac{1}{1-q} \operatorname{ch}(U), \operatorname{ch} U\left[t^{-1}\right]=\frac{1}{1-q^{-1}} \operatorname{ch} U ;
$$

(co)cyclicity of $U$ implies (co)cyclicity of $\left(U\left[t^{-1}\right]\right) U[t]$;

$$
\left(U^{*}\right)\left[t^{-1}\right]=(U[t])^{*} \text {. }
$$

We consider the vector space $U\left[t, t^{-1}\right]:=U \otimes \mathbb{C}\left[t, t^{-1}\right]$ and define the following action of $\mathfrak{g}[t]$ on this space: for any $m \in \mathbb{Z}_{\geq 0}, k \in \mathbb{Z}, x \in \mathfrak{g}, u \in U$ :

$$
x t^{m} \cdot u \otimes t^{k}=\sum_{j=0}^{m}\left(\begin{array}{c}
m \\
j
\end{array}\right)\left(x t^{j} u\right) \otimes t^{m+k-j} .
$$

It is indeed an action:

$$
\begin{gathered}
\left(y t^{n} x t^{m}-x t^{m} y t^{n}\right) \cdot u \otimes t^{k}=\sum_{i=0}^{n}\left(\begin{array}{c}
n \\
i
\end{array}\right) \sum_{j=0}^{m}\left(\begin{array}{c}
m \\
j
\end{array}\right)\left(y t^{i} x t^{j} u-x t^{j} y t^{i} u\right) \otimes t^{m+n+k-i-j} \\
=\sum_{i, j=0}^{\infty}\left(\begin{array}{c}
m \\
j
\end{array}\right)\left(\begin{array}{c}
n \\
i
\end{array}\right)\left([x, y] t^{i+j} u\right) \otimes t^{m+n+k-i-j}=[x, y] t^{m+n} \cdot u \otimes t^{k} .
\end{gathered}
$$

The last equality holds because $\sum_{i+j=c}\left(\begin{array}{c}m \\ j\end{array}\right)\left(\begin{array}{c}n \\ i\end{array}\right)=\left(\begin{array}{c}m+n \\ c\end{array}\right)$.

Definition 1.2. We define $U[t]$ as a submodule $U \otimes \mathbb{C}[t]$ of $U\left[t, t^{-1}\right]$. We define $U\left[t^{-1}\right]$ as a quotient mdoule $U\left[t, t^{-1}\right] / U \otimes t \mathbb{C}[t]$ of $U\left[t, t^{-1}\right]$.

Lemma 1.3. Properties (1.2), (1.3), (1.4) hold.

Proof. The equations on the characters are obvious. To show cyclicity and cocyclicity it suffices to apply standard linear algebra arguments (the Vandermonde determinant). Finally, define the pairing between $U[t]$ and $U^{*}\left[t^{-1}\right]$ in the following way:

$$
\left(u_{1} \otimes t^{k}, u_{2} \otimes t^{l}\right)=\delta_{k+l, 0}\left(u_{1}, u_{2}\right) .
$$

This pairing gives a duality of modules.

Proposition 1.4. Let $W_{\omega_{i}}$ be the fundamental local Weyl module. Then $W_{\omega_{i}}[t] \simeq \mathbb{W}_{\omega_{i}}, W_{\omega_{i}}^{*}\left[t^{-1}\right] \simeq\left(\mathbb{W}_{\omega_{i}}\right)^{*}$.

Proof. $W_{\omega_{i}}[t]$ is the cyclic module satisfying all defining relations of the global Weyl module and has the same character. Therefore these modules are isomorphic. The second claim follows from (1.4). 
1.4. Semi-infinite flag varieties. We consider the product of projective spaces $\prod_{\lambda \in P_{+}} \mathbb{P}\left(V_{\lambda}[[t]]\right)$. The semi-infinite fag variety $\mathfrak{Q}$ is a subvariety of this product formed by all collections of lines $L_{\lambda} \subset V_{\lambda}[[t]]$ such that $L_{\lambda+\mu} \mapsto L_{\lambda} \otimes L_{\mu}$ under the natural embedding $V_{\lambda+\mu}[[t]] \rightarrow V_{\lambda}[[t]] \otimes_{\mathbb{C}[[t]]} V_{\mu}[[t]]$ ([FMi, $[\mathrm{BG}])$. One easily sees that $\mathfrak{Q}$ is embedded into $\prod_{i=1}^{r} \mathbb{P}\left(V_{\omega_{i}}[[t]]\right)$. This embedding gives rise to a family of line bundles $\mathcal{O}(\lambda)$ on $\mathfrak{Q}$. The following theorem is proved in [BF1, Kat]:

$$
H^{0}(\mathfrak{Q}, \mathcal{O}(\lambda)) \simeq \mathbb{W}_{\lambda}^{*} .
$$

We denote the projective coordinate ring of $\mathfrak{Q}$ by $\mathbb{W}^{*}=\bigoplus_{\lambda \in P_{+}} \mathbb{W}_{\lambda}^{*}$.

1.5. Integrable representations. Let $\widehat{\mathfrak{g}}=\mathfrak{g} \otimes \mathbb{C}\left[t, t^{-1}\right] \oplus \mathbb{C} K \oplus \mathbb{C} d$ be the affine Kac-Moody Lie algebra (here $K$ is central and $d$ satisfies $\left[d, x \otimes t^{i}\right]=$ $\left.-i x \otimes t^{i}\right)$. The Cartan subalgebra $\mathfrak{h}^{a}$ of $\widehat{\mathfrak{g}}$ is equal to $\mathfrak{h} \otimes 1 \oplus \mathbb{C} K \oplus \mathbb{C} d$. Let $\delta \in\left(\mathfrak{h}^{a}\right)^{*}$ be the basic imaginary root. For an affine weight we define its finite part as the projection to $\mathfrak{h}^{*}$.

Let $\Lambda_{0}, \ldots, \Lambda_{d}$ be the level one integrable weights (i.e. $\Lambda_{i}(K)=1$ ). In what follows we use the fact that $d+1=|P / Q|$. Let $W^{a}$ be the affine Weyl group (the Weyl group of $\widehat{\mathfrak{g}}$ ) and let $L\left(\Lambda_{i}\right)$ be the integrable level one representations of $\widehat{\mathfrak{g}}$. Let

$$
\mathbb{L}=\bigoplus_{i=0}^{d} L\left(\Lambda_{i}\right) .
$$

For any integral weight $\lambda \in P$ there exists an element $w \in W^{a}$ and $0 \leq i \leq d$ such that the finite part of $w \Lambda_{i}$ is equal to $\lambda$. In other words, for any $\lambda \in P$ there exists extremal vector $u_{\lambda} \in \mathbb{L}$ of finite weight $\lambda$. Following $\mathrm{KL}$ we introduce the notation $\left(\lambda \in P_{+}\right)$:

$$
F_{\lambda}=\mathrm{U}\left(\mathfrak{g} \otimes \mathbb{C}\left[t^{-1}\right]\right) u_{\lambda} \subset \mathbb{L} .
$$

In particular, each $F_{\lambda}$ is $\mathfrak{g} \otimes \mathbb{C}\left[t^{-1}\right]$ module and $F_{\lambda} \supset F_{\mu}$ if $\lambda<\mu$. The following statement is proved in $[\mathrm{KL}]$ :

$$
\frac{F_{\lambda}}{\sum_{\mu>\lambda} F_{\mu}} \simeq \mathbb{W}_{\lambda} .
$$

The isomorphism (1.9) is the isomorphism of $\mathfrak{g} \otimes \mathbb{C}\left[t^{-1}\right]$ modules, where $\mathbb{W}_{\lambda}$ is made into $\mathfrak{g} \otimes \mathbb{C}\left[t^{-1}\right]$ module by the substitution $t \mapsto t^{-1}$.

Let $\sigma$ be Chevalley antiautomorphism on $\widehat{\mathfrak{g}}$ defined by

$$
\begin{gathered}
\sigma\left(e_{\alpha} \otimes t^{k}\right)=f_{-\alpha} \otimes t^{-k}, \alpha \in \Delta ; \\
\sigma\left(h \otimes t^{k}\right)=h \otimes t^{-k}, h \in \mathfrak{h} .
\end{gathered}
$$

We consider the Shapovalov bilinear form $(\cdot, \cdot): L\left(\Lambda_{i}\right) \otimes L\left(\Lambda_{i}\right) \rightarrow \mathbb{C}$ with the property $(x \cdot u, v)=(u, \sigma(x) \cdot v)$ for all $x \in \widehat{\mathfrak{g}}$. 
Definition 1.5. We define the increasing filtration on $L\left(\Lambda_{i}\right)$ by subspaces

$$
F_{\lambda}^{\dagger}=\bigcap_{\mu>\lambda^{*}} \operatorname{ann} F_{\mu}, \lambda \in P_{+}
$$

where $\operatorname{ann} F_{\mu}$ is the orthogonal complement to $F_{\mu}$ with respect to the Shapovalov form.

Remark 1.6. The notation for $F_{\lambda}^{\dagger}$ is chosen in such a way that $u_{\lambda} \in F_{\lambda}^{\dagger}$. In fact, the Shapovalov form pairs nontrivially $u_{\lambda}$ and $u_{-\lambda}$. The vector $u_{-\lambda}$ is the lowest weight vector of $\mathfrak{g}$-module, whose highest weight vector is $u_{-w_{0} \lambda}=u_{\lambda^{*}}$. We conclude that if $\mu>\lambda^{*}$, then $u_{\lambda} \in \operatorname{ann} F_{\mu}$.

Note that each $F_{\lambda}^{\dagger}$ is a $\mathfrak{g}[t]$-module (since $F_{\lambda}$ is $\mathfrak{g}\left[t^{-1}\right]$-invariant).

Proposition 1.7. We have isomorphism of $\mathfrak{g}[t]$-modules

$$
F_{\lambda}^{\dagger} / \sum_{\nu<\lambda} F_{\nu}^{\dagger} \simeq \mathbb{W}_{\lambda^{*}}^{*}
$$

Proof. It suffices to show that

$$
F_{\lambda}^{\dagger} / \sum_{\nu<\lambda} F_{\nu}^{\dagger}=\left(F_{\lambda^{*}} / \sum_{\mu>\lambda^{*}} F_{\mu}\right)^{*}
$$

We have a pairing $(\cdot, \cdot): F_{\lambda^{*}} \otimes F_{\lambda}^{\dagger} \rightarrow \mathbb{C}$ and $\sum_{\mu>\lambda^{*}} F_{\mu}$ is the annihilator of $F_{\lambda}^{\dagger}, \sum_{\nu<\lambda} F_{\nu}^{\dagger}$ is the annihilator of $F_{\lambda^{*}}$.

In what follows fro a filtration $F_{\lambda}$ we use the notation $F<\lambda=\sum_{\nu<\lambda} F_{\nu}$ and similarly for $F_{\leq \lambda}$.

We have the following characterization of $\mathfrak{g}[t]$-modules $F_{\lambda}^{\dagger}$ which can be immediately obtained from the duality.

Proposition 1.8. Let us fix $i$ such that $u_{\lambda} \in L\left(\Lambda_{i}\right)$. Then $F_{\lambda}^{\dagger}$ consists of vectors $v \in L\left(\Lambda_{i}\right)$ such that all (finite) $\mathfrak{h}$-weights of elements $\mathrm{U}(\mathfrak{g}[t]) v$ are less than or equal to $\lambda$.

Proof. Assume that for some $x \in \mathrm{U}(\mathfrak{g}[t])$ the weight $\mu$ of the element $x v$ is not less than or equal to $\lambda$. Then (since $L\left(\Lambda_{i}\right)$ is of level one) for some element $y \in \mathrm{U}(\mathfrak{h}[t])$ we have

$$
y x v=u_{\mu} .
$$

Thus $u_{\mu} \in F_{\lambda}^{\dagger}$, which implies $\mu \leq \lambda$ (see Remark 1.6).

\section{Generalities on T-GRAded Vertex Algebras}

2.1. Definition and basic properties. In this section we recall the general concept of $\Gamma$-graded vertex algebras following $[\mathrm{R}, \mathrm{DL}]$.

Remark 2.1. We only use the $\Gamma$-graded vertex algebras of weight lattices with $\Gamma=P / Q$ (weight lattice modulo root lattice). For example, in the $E_{8}$-case $\Gamma$ is trivial and we obtain the usual vertex algebra. 
Let $\Gamma$ be a finite abelian group, $N$ be exponent of $\Gamma$, i. e. the smallest integer such that $N \Gamma=\{0\}$. Let $\Delta$ be a $\mathbb{Z}$-bilinear symmetric map $\Gamma \times \Gamma \rightarrow$ $\mathbb{Q} / \mathbb{Z}$ and $\nu: \Gamma \times \Gamma \rightarrow \mathbb{C}^{*}$ be a map satisfying

$$
\nu(g, h) \nu(h, g)=e^{-2 \pi i \Delta(g, h)} \forall g, h \in \Gamma ; \nu(g, g)=e^{-\pi i \Delta(g, g)} .
$$

For a $\Gamma$-graded vector space $V=\bigoplus_{g \in \Gamma} V_{g}$ the algebra $\operatorname{End}(V)$ is $\Gamma$-graded in the usual way. An element $X(z)=\sum_{n \in \frac{1}{N} \mathbb{Z}} x_{(n)} z^{-1-n} \in \operatorname{End}(V)\left[\left[z^{ \pm 1 / N}\right]\right]$ is called a generalized vertex operator if for any $v \in V x_{(n)} v=0$ for $n>>0$. The vertex operator $X(z)$ is called homogeneous of parity $g$ if all $x_{(n)} \in$ $\operatorname{End}(V)_{g}$ (i. e. $x_{(n)} V_{h} \subset V_{h+g}$ for any $h \in \Gamma$ ) and for all $v \in V_{h} x_{(n)} v=0$ unless $n \in \Delta(g, h)$. The parity of a homogeneous element $a$ is denoted by $p(a)$.

For an arbitrary rational $n$ denote by $i_{z, w}(z-w)^{n}$ the formal expansion of $(z-w)^{n}$ in the domain $|w|<|z|$ :

$$
i_{z, w}(z-w)^{n}:=\sum_{j=0}^{\infty}(-1)^{j}\left(\begin{array}{l}
n \\
j
\end{array}\right) z^{n-j} w^{j},\left(\begin{array}{l}
n \\
j
\end{array}\right)=\frac{n(n-1) \ldots(n-j+1)}{j !} .
$$

Analogously denote by $i_{w, z}(z-w)^{n}$ the formal expansion of $(z-w)^{n}$ in domain the $|z|<|w|:$

$$
i_{w, z}(z-w)^{n}:=e^{i n \pi} \sum_{j=0}^{\infty}(-1)^{j}\left(\begin{array}{l}
n \\
j
\end{array}\right) w^{n-j} z^{j} .
$$

Definition 2.2. Two homogeneous generalized vertex operators $X(z), Y(z)$ of degrees $g$ and $h$ are mutually local with respect to $\Delta$ and $\nu$ if for large enough $n \in \Delta(g, h)$ :

$$
i_{z, w}(z-w)^{n} X(z) Y(w)=i_{w, z}(z-w)^{n} \nu(g, h) Y(w) X(z) .
$$

Definition 2.3. For two homogeneous vertex operators $A(z)=\sum a_{(n)} z^{-1-n}$, $B(z)$ define their normally ordered product : $A(z) B(z):$ by

$$
: A(z) B(z):=\sum_{n<0} a_{(n)} z^{-1-n} B(z)+B(z) \sum_{n \geq 0} a_{(n)} z^{-1-n} .
$$

Definition 2.4. A $\Gamma$-graded vertex algebra is a vector space $V=\bigoplus_{g \in \Gamma} V_{g}$ with the following data:

(i) a vacuum vector $|0\rangle \in V_{0}$;

(ii) a linear operator $T$ (translation operator);

(iii) generalized vertex operators labeled by $a \in V$

$$
Y(a, z)=\sum_{n \in \frac{1}{N} \mathbb{Z}} a_{(n)} z^{-1-n} \in \operatorname{End}(V)\left[\left[z^{1 / N}, z^{-1 / N}\right]\right]
$$

satisfying the following properties

(0) the operation $Y(a, z)$ is linear in a and for a homogeneous $a \in V_{g}$ has parity $g$;

(1) (Translation) $[T, Y(a, z)]=\frac{\partial}{\partial z} Y(a, z)$ and $T|0\rangle=0$; 
(2) (Vacuum) $Y(|0\rangle, z)=\operatorname{Id}_{V}, Y(a, z)|0\rangle \in \operatorname{End}(V)\left[\left[z^{1 / N}\right], Y(a, 0)|0\rangle=a\right.$;

(3) (Locality) For all homogeneous $a, b \in V$ the operators $Y(a, z)$ and $Y(b, z)$ are mutually local with respect to $\Delta$ and $\nu$.

We need the following property of $\Gamma$-graded vertex algebras (see $[\mathbb{R}$, Theorem 4.1.25).

Proposition 2.5. For any homogenous elements $a, b, c \in V$, any $n \in$ $\Delta(p(a), p(b)), m \in \Delta(p(a), p(c)), k \in \Delta(p(b), p(c))$ :

$$
\begin{aligned}
& \sum_{j=0}^{\infty}\left(\begin{array}{c}
m \\
j
\end{array}\right)\left(a_{(n+j)} b\right)_{(m+k-j)} c \\
= & \sum_{j=0}^{\infty}(-1)^{j}\left(\begin{array}{c}
n \\
j
\end{array}\right)\left(a_{(n+m-j)} b_{(k+j)}-e^{n \pi i} \nu(p(a), p(b)) b_{(n+k-j)} a_{(m+j)}\right) c .
\end{aligned}
$$

We need the following corollary.

Corollary 2.6. For any $a \in V_{0}, b, c \in V, m \in \mathbb{Z}, k \in \Delta(p(b), p(c))$ :

$$
\left[a_{(m)}, b_{(k)}\right] c=\sum_{j=0}^{\infty}\left(\begin{array}{c}
m \\
j
\end{array}\right)\left(a_{(j)} b\right)_{(m+k-j)} c .
$$

Proof. This is a particular case of (2.1) for $n=0$.

Corollary 2.7. Let $n \in \Delta(p(a), p(b))$ satisfies $a_{(n+j)} b=0$ for any integer $j \geq 0$. Then:

$$
i_{z, w}(z-w)^{n} Y(a, z) Y(b, w)=i_{w, z}(z-w)^{n} \nu(p(a), p(b)) Y(b, w) Y(a, z) .
$$

Proof. Due to equation (2.2) the difference between the coefficients of left and right hand sides of this formula applied to an element $c \in V$ is equal to

$$
\sum_{j=0}^{\infty}\left(\begin{array}{c}
m \\
j
\end{array}\right)\left(a_{(n+j)} b\right)_{(m+k-j)} c
$$

which vanishes.

2.2. Lattice $\Gamma$-graded vertex algebras. Let $P$ be a lattice, i .e. a finitely generated free abelian group with a bilinear map $(\cdot, \cdot): P \times P \rightarrow \mathbb{Q}$. Let $Q \subset P$ be an even integral sublattice of the same rank as $P$ such that $(Q, P) \subset \mathbb{Z}$. Let $\Gamma=P / Q, \Gamma=\left\{g_{0}, g_{1}, \ldots, g_{d}\right\}$.

We denote by $p$ the natural projection $P \rightarrow \Gamma$. For an element $g_{i} \in \Gamma$ we fix its representative $\chi_{i} \in P$. Define the $\mathbb{Z}$-bilinear map $\Gamma \times \Gamma \rightarrow \mathbb{Q} / \mathbb{Z}$ :

$$
\Delta\left(g_{i}, g_{j}\right):=\mathbb{Z}-\left(\chi_{i}, \chi_{j}\right) .
$$

It is easy to see that such a form does not depend on a choice of representatives. Next we define the following map $\Gamma \times \Gamma \rightarrow \mathbb{C}^{*}$ :

$$
\nu\left(g_{i}, g_{j}\right):=e^{i \pi\left(\chi_{i}, \chi_{j}\right)}
$$


and the function $B: P \times P \rightarrow \mathbb{C}^{*}$ :

$$
B(\lambda, \mu)=e^{-i \pi(\lambda, \mu)} \nu(p(\lambda), p(\mu)) .
$$

Note that $B(P \times P) \subset\{ \pm 1\}$. We fix a cocycle $\epsilon$ on $P$ with the following property:

$$
\epsilon\left(\omega_{i}, \omega_{j}\right)= \begin{cases}1, & \text { if } i \leq j \\ B\left(\omega_{i}, \omega_{j}\right), & \text { if } i>j\end{cases}
$$

Such a 2-cocycle exists (see $[\underline{S}, \overline{D L}$ ) and defines a new structure of an associative algebra on the space $\mathbb{C}[P]$ :

$$
e^{\lambda} e^{\mu}=\epsilon(\lambda, \mu) e^{\lambda+\mu}
$$

This structure is obtained from the projective representation of $P$ on its group algebra constructed via the cocycle $\epsilon$.

Our next task is to define the space of states of our VOA. Let $\mathfrak{h}=\mathbb{C} \otimes_{\mathbb{Z}} P$. The form $(\cdot, \cdot)$ can be extended to $\mathfrak{h}$ by linearity. Consider the vector space $\mathfrak{h}\left[t, t^{-1}\right]$ of formal Laurent polynomials in $t$ with coefficients in $\mathfrak{h}$. For $h \in \mathfrak{h}$, $k \in \mathbb{Z}$ we set $h t^{k}=h \otimes t^{k}$. Let $\widehat{\mathfrak{h}}=\mathfrak{h}\left[t, t^{-1}\right] \oplus \mathbb{C} c$ be the Heisenberg algebra, i.e. the Lie algebra with the following bracket:

$$
\left[h_{1} t^{k}, h_{2} t^{l}\right]=k \delta_{k+l, 0}\left(h_{1}, h_{2}\right) c
$$

and $c$ is a central element. Then the Fock (Verma) module of $\widehat{\mathfrak{h}}$ is the module $U(\widehat{\mathfrak{h}}) /(U(\widehat{\mathfrak{h}}) \mathfrak{h}[t]+U(\widehat{\mathfrak{h}})(c-1))$.

Consider the vector space $t^{-1} \mathfrak{h}\left[t^{-1}\right]$ of formal polynomials in $t^{-1}$ with coefficients in $\mathfrak{h}$ without zero term and let $\mathbb{L}=S\left(t^{-1} \mathfrak{h}\left[t^{-1}\right]\right) \otimes \mathbb{C}[P]$. This space has a structure of an associative algebra. Let $T$ be the following derivation of this algebra:

$$
T\left(h t^{-n} \otimes 1\right)=n\left(h t^{-n-1} \otimes 1\right), T\left(1 \otimes e^{\lambda}\right)=\lambda t^{-1} \otimes e^{\lambda}, h \in \mathfrak{h}, \lambda \in P .
$$

Let $|0\rangle:=1 \otimes e^{0}$ be the vacuum vector. We identify $S\left(t^{-1} \mathfrak{h}\left[t^{-1}\right]\right)$ with the Fock space.

For an element $h \in \mathfrak{h}$ we define the following generalized vertex operators

$$
\begin{gathered}
Y\left(h t^{-1} \otimes 1, z\right)=\sum_{k \in \mathbb{Z}} h t^{k} z^{-1-k}, \\
Y\left(h t^{-1-n} \otimes 1, z\right)=\frac{1}{n !} \frac{\partial^{n}}{\partial z^{n}} Y\left(h t^{-1}, z\right) .
\end{gathered}
$$

Then define

$$
Y\left(1 \otimes e^{\lambda}, z\right)=\exp \left(\sum_{n>0} \frac{\lambda t^{-n}}{n} z^{n}\right) \exp \left(\sum_{n<0} \frac{\lambda t^{-n}}{n} z^{n}\right) \otimes e^{\lambda} z^{\lambda},
$$

where $z^{\lambda} e^{\mu}=e^{\mu} z^{(\lambda, \mu)}$. Finally, for any $a \in t^{-1} \mathfrak{h}\left[t^{-1}\right], b \in \mathbb{L}$

$$
Y(a b, z)=: Y(a, z) Y(b, z): \text {. }
$$


2.3. Lie algebra action. Let $\mathbb{L}$ be $\Gamma$-graded lattice vertex algebra of the positively defined lattice $P$. Let $\left\{\alpha_{i}\right\},\left\{\omega_{i}\right\}$ be dual bases of $\mathfrak{h}$. Consider the element $\omega=\sum_{i=1}^{r} \alpha_{i} t^{-1} \cdot \omega_{i} t^{-1} \otimes e^{0}$ (the conformal vector). Then the operator $\omega_{(1)}$ is diagonalizable and an element $\prod_{i=1}^{n} h_{i} t^{-s_{i}} \otimes e^{\lambda}$ is an eigenvector with the eigenvalue $\frac{(\lambda, \lambda)}{2}+\sum_{i=1}^{n} s_{i}$ (see, for example, $[\mathrm{R}$, Chapter 4.3). This eigenvalue is called the conformal weight. Let $\mathbb{L}[i]$ be the space of elements of conformal weight $i$.

Proposition 2.8. The operators $A_{(n)}, A \in \mathbb{L}[1], n \in \mathbb{Z}$ form an untwisted affine Lie algebra corresponding to the finite root system $\{\alpha \in P \mid(\alpha, \alpha)=2\}$, $e_{\alpha} t^{n} \mapsto\left(1 \otimes e^{\alpha}\right)_{(n)}, h_{\alpha} t^{n} \mapsto\left(\alpha t^{-1} \otimes e^{0}\right)_{(n)}$. If $P$ is a weight lattice of the simple Lie algebra $\mathfrak{g}$, then for each $g \in P / Q \mathbb{L}_{g}$ is a level-one integrable representation of $\widehat{\mathfrak{g}}$.

Proof. The first claim can be easily seen from formula (2.6). The second claim is contained in [FK].

We close this section with an example.

Example 2.9. Let $\mathfrak{g}=\mathfrak{s l}_{2}$. Then $\Gamma=\mathbb{Z} / 2 \mathbb{Z}=\{e, g\}$. One has

$$
\begin{gathered}
\Delta(e, e)=\Delta(e, g)=\Delta(g, e)=\mathbb{Z}, \Delta(g, g)=-1 / 2+\mathbb{Z}, \\
\nu(e, e)=\nu(e, g)=\nu(g, e)=1, \nu(g, g)=e^{i \pi / 2} .
\end{gathered}
$$

The space of states is given by $\mathbb{L}=L\left(\Lambda_{0}\right) \oplus L\left(\Lambda_{1}\right)$. For vectors $e^{ \pm \omega}=$ $1 \otimes e^{ \pm \omega} \in L\left(\Lambda_{1}\right)$ one gets

$$
\begin{gathered}
\left.Y\left(e^{ \pm \omega}, z\right)\right|_{L\left(\Lambda_{0}\right)}=\sum_{n \in \mathbb{Z}} e_{(n)}^{ \pm \omega} z^{-n-1}, \\
\left.Y\left(e^{ \pm \omega}, z\right)\right|_{L\left(\Lambda_{1}\right)}=\sum_{n \in-1 / 2+\mathbb{Z}} e_{(n)}^{ \pm \omega} z^{-n-1} .
\end{gathered}
$$

Finally, the conformal weight of $e^{ \pm \omega}$ is equal to $1 / 4$.

\section{The STRATEGY}

Recall the space $\mathbb{L}=\bigoplus_{i=0}^{d} L\left(\Lambda_{i}\right)$, the direct sum of all level one integrable $\widehat{\mathfrak{g}}$ modules. The space $\mathbb{L}$ carries the structure of $P / Q$-graded vertex operator algebra.

Remark 3.1. If $\mathfrak{g}$ is of type $A_{n}$, then $d=n$. If $\mathfrak{g}$ is of type $E_{8}$, then $d=0$ (i.e. the only level one integrable module is the basic one, $L\left(\Lambda_{0}\right)$ ).

Recall that $\mathbb{L}$ is filtered by the (increasing) filtration $F_{\lambda}^{\dagger}$ and according to Proposition 1.7 the associated graded space is isomorphic to the direct sum of dual global Weyl modules $\mathbb{W}^{*}=\bigoplus_{\lambda \in P_{+}} \mathbb{W}_{\lambda}^{*}$. This direct sum $\mathbb{W}^{*}$ carries natural structure of commutative associative algebra, where the multiplication is induced by the natural embeddings $\mathbb{W}_{\lambda+\mu} \hookrightarrow \mathbb{W}_{\lambda} \otimes \mathbb{W}_{\mu}$ (see [Kat]). Our goals are as follows: 
- to describe the filtration $F_{\lambda}^{\dagger}$ in terms of the VOA structure on $\mathbb{L}$;

- to describe the multiplication in $\mathbb{W}^{*}$ in terms of the VOA $\mathbb{L}$;

- to endow the space $\mathbb{W}^{*}$ with the structure of vertex operator algebra;

- to derive (conjecturally, defining) relations in $\mathbb{W}^{*}$ using the VOA structure.

Our strategy is as follows. We define an increasing filtration $G_{\lambda} \subset \mathbb{L}$, $\lambda \in P_{+}$using the action of vertex operators. In short, the definition works as follows. We have natural projection from global to local Weyl modules $\mathbb{W}_{\lambda} \rightarrow W_{\lambda}$, which induce the embeddings $W_{\lambda}^{*} \subset \mathbb{W}_{\lambda}^{*}$. For each $i=1, \ldots, r$ we fix a lift $\widetilde{W}_{\omega_{i}^{*}}^{*} \subset F_{\omega_{i}}^{\dagger}$ (recall that $F_{\lambda}^{\dagger} / F_{<\lambda}^{\dagger} \simeq \mathbb{W}_{\lambda^{*}}^{*}$ ). Then $G_{\lambda}$ is a linear span of the monomials of the form

$$
\tilde{A}_{\left(m_{1}\right)}^{(1)} \ldots \tilde{A}_{\left(m_{s}\right)}^{(s)}|0\rangle
$$

where $\tilde{A}^{l}$ are elements of $\widetilde{W}_{\omega_{i}^{*}}^{*}$ and for each $i=1, \ldots, r$ there are exactly $\left(\lambda, \alpha_{i}^{\vee}\right)$ vectors $\tilde{A}^{l}$ from $\widetilde{W}_{\omega_{i}^{*}}^{*}$. We first prove that

- $A_{(n)} B \in G_{\lambda+\mu}^{\dagger}$ for all $A \in G_{\lambda}^{\dagger}, B \in G_{\mu}^{\dagger}, n \in \frac{1}{N} \mathbb{Z}$;

- $A_{(n)} B \in F_{\lambda+\mu}^{\dagger}$ for all $A \in F_{\lambda}^{\dagger}, B \in F_{\mu}^{\dagger}, n \in \frac{1}{N} \mathbb{Z}$;

- $G_{\lambda} \subset F_{\lambda}^{\dagger}$ for all $\lambda \in P_{+}$.

Example 3.2. Let $\mathfrak{g}=\mathfrak{s l}_{2}$. In the notation of Example 2.9

$$
G_{m \omega}=\operatorname{span}\left\{e_{\left(n_{1}\right)}^{ \pm \omega} \ldots e_{\left(n_{m}\right)}^{ \pm \omega}|0\rangle, n_{i} \in \mathbb{Z}\right\} .
$$

Remark 3.3. If $\mathfrak{g}$ is of type $A_{n}$, then $\sum_{\lambda<\omega_{i}} F_{\lambda}^{\dagger}=0$ for all $i$ (since $\left.\left\{\lambda \in P_{+}, \lambda<\omega_{i}\right\}=\emptyset\right)$ and $F_{\omega_{i}}^{\dagger} \subset L\left(\Lambda_{i}\right)$ for $i=1, \ldots, n$. In particular, $\left(\operatorname{gr} F^{\dagger}\right)_{\omega_{i}}=F_{\omega_{i}}^{\dagger} \simeq \mathbb{W}_{\omega_{i}^{*}}^{*}$. However, this is not true in types $D$ and $E$. For example, in type $E_{8}$ all the spaces $F_{\omega_{i}}^{\dagger}$ belong to the same (the only) integrable level one $\widehat{\mathfrak{g}}$ module.

Recall the lifts $\widetilde{W}_{\omega_{i}^{*}}^{*} \subset F_{\omega_{i}}^{\dagger}$. By Proposition 1.4 we have an isomorphism of $\mathfrak{g}[t]$-modules

$$
\left(\operatorname{gr} F^{\dagger}\right)_{\omega_{i}} \simeq W_{\omega_{i}^{*}}^{*}\left[t^{-1}\right] .
$$

In order to write down the multiplication in the algebra $\mathbb{W}^{*}=\bigoplus_{\lambda \in P_{+}} \mathbb{W}_{\lambda}^{*}$ we proceed as follows. Let $\lambda=\omega_{j_{1}}+\cdots+\omega_{j_{s}}$ and let us consider a collection of elements $A^{k} \in W_{\omega_{j_{k}}^{*}}^{*}$ and their lifts $\tilde{A}^{k} \in \widetilde{W}_{\omega_{j_{k}}^{*}}^{*}, k=1, \ldots, s$ and collection of nonnegative integers $m_{1}, \ldots, m_{s}$. We write down a formula for the product of the elements $A^{k} \otimes t^{-m_{k}} \in \mathbb{W}_{\omega_{j_{k}}^{*}}^{*}$. Consider the expression

$$
\mathcal{M}\left(\tilde{A}^{1}, \ldots, \tilde{A}^{s}\right)=\prod_{1 \leq k<l \leq s} i_{z_{k}, z_{l}}\left(z_{k}-z_{l}\right)^{-\left(\omega_{i_{k}}^{*}, \omega_{i_{l}}^{*}\right)} \prod_{k=1}^{s} Y\left(\tilde{A}^{k}, z_{k}\right)|0\rangle .
$$


Here we use the notation $\prod_{i=1}^{s} X_{i}=X_{1} \ldots X_{s}$ for possible noncommuting $X_{i}$. We denote by $\mathcal{M}\left(\tilde{A}^{1}, \ldots, \tilde{A}^{s}\right)_{m_{1}, \ldots, m_{s}}$ the coefficient of $z_{1}^{-m_{1}} \ldots z_{s}^{-m_{s}}$ in $\mathcal{M}\left(\tilde{A}^{1}, \ldots, \tilde{A}^{s}\right)$. We show that the multiplication rule

$$
A^{1} \otimes t^{-m_{1}} \ldots A^{s} \otimes t^{-m_{s}}=\mathcal{M}\left(\tilde{A}^{1}, \ldots, \tilde{A}^{s}\right)_{m_{1}, \ldots, m_{s}}
$$

induces the map

$$
\varphi: \mathbb{W}_{\omega_{j_{1}}^{*}}^{*} \otimes \ldots \otimes \mathbb{W}_{\omega_{j_{s}}^{*}}^{*} \rightarrow G_{\lambda} /\left(G_{\lambda} \cap F_{<\lambda}^{\dagger}\right) .
$$

We then prove that

- $\varphi$ does not depend on the choice of lifts $\widetilde{W}_{\omega_{j_{k}}^{*}}^{*}$;

- $\varphi$ is commutative;

- $\varphi$ is a homomorphism of $\mathfrak{g}[t]$-modules.

From this we derive that

- $G_{\lambda}=F_{\lambda}^{\dagger}$ for all $\lambda \in P_{+}$;

- the multiplication (3.2) coincides with the multiplication in $\mathbb{W}^{*}$.

Remark 3.4. The $P / Q$-graded vertex operator algebra structure on $\mathbb{W}^{*}$ comes from the property $A_{(n)} B \in G_{\lambda+\mu}$ for all $A \in G_{\lambda}, B \in G_{\mu}$ and $n \in \frac{1}{N} \mathbb{Z}$.

Now let $A \in W_{\omega_{i}^{*}}^{*}, B \in W_{\omega_{j}^{*}}^{*}$ and let us consider $A$ and $B$ as elements of $P / Q$-graded VOA $\mathbb{W}^{*}$ (via the embedding $W_{\omega_{i}^{*}}^{*} \subset \mathbb{W}_{\omega_{i}^{*}}^{*}$ and Remark 3.4). Let $m_{i, j}=-\left(\omega_{i}^{*}, \omega_{j}^{*}\right)$. We show that $\left(A_{\left(m_{i, j}-s\right)} B\right)_{(-1-r)}|0\rangle$ is equal to the coefficient of $z^{r}$ in

$$
\left.\left(\partial_{z}^{s-1} i_{z, w}(z-w)^{m_{i, j}} Y(A, z) Y(B, w)\right)\right|_{z=w}|0\rangle .
$$

This observation produces a set of quadratic relations for the Plücker-Drinfeld embedding of the semi-infinite flag varieties. We conjecture that this is a defining set of relations and prove the conjecture in type $A$.

\section{VOA FILTRATION AND MULTIPLICATION FORMULA}

4.1. VOA filtration. Let us introduce the increasing filtration on $\mathbb{L}$ which should be understood as the $\Gamma$-graded analogue of the so-called standard filtration (see [A, Li1, Li2]). The filtration spaces $G_{\lambda} \subset \mathbb{L}$ are labeled by the dominant integer weights $\lambda \in P_{+}$. The filtration is defined inductively as follows:

$$
G_{0}=\mathbb{C}|0\rangle, G_{\lambda}=\sum_{\mu<\lambda} G_{\mu}+\sum_{i: \lambda-\omega_{i} \in P_{+}} \operatorname{span}\left\{B_{(k)} G_{\lambda-\omega_{i}}, B \in \widetilde{W}_{\omega_{i}^{*}}^{*}, k \in \frac{1}{N} \mathbb{Z}\right\} .
$$

Remark 4.1. Let $\mathfrak{g}$ be of type $A_{n}$. Then $W_{\omega_{i}^{*}}^{*} \simeq V_{\omega_{i}^{*}}^{*}=F_{\omega_{i}}^{\dagger}$ is the top of the integrable $\widehat{\mathfrak{g}}$-module $L\left(\Lambda_{i}\right)$.

For a subspace $U \subset \mathbb{L}$ we use the notation $U_{(n)}=\left\{u_{(n)}: u \in U\right\}$.

Proposition 4.2. For any $n \in \frac{1}{N} \mathbb{Z}:\left(G_{\lambda}\right)_{(n)} G_{\mu} \subset G_{\lambda+\mu}$. 
Proof. We prove Proposition by induction on $\lambda$. The basis of induction is the trivial case $\lambda=0$. Assume first that $\lambda=\omega_{i}$ is the fundamental weight. Then for $A \in \widetilde{W}_{\omega_{i}^{*}}^{*}$ we need to prove the following:

$$
\left(A_{(k)}|0\rangle\right)_{(n)} G_{\mu} \subset G_{\mu+\omega_{i}} .
$$

However the operators $\left(A_{(k)}|0\rangle\right)_{(n)}$ are scalar multiples of operators $A_{(k+n+1)}$. Therefore Proposition holds by definition of $G_{\mu}$.

For general $\lambda$ take an element $A_{(k)} B, A \in{\widetilde{W^{*}}}_{i}^{*}, B \in G_{\lambda-\omega_{i}}$. Then using equation (2.5) we obtain that for any $C$ :

$$
\left(A_{(k)} B\right)_{(n)} C \in \sum_{i \in \mathbb{Z}} \mathbb{C} A_{(k+i)}\left(B_{(n-i)} C\right)+\sum_{i \in \mathbb{Z}} \mathbb{C} B_{(n-i)}\left(A_{(k+i)} C\right) .
$$

So the proof is completed by induction.

Proposition 4.3. For any $n \in \frac{1}{N} \mathbb{Z}$ :

$$
\left(F_{\lambda}^{\dagger}\right)_{(n)} F_{\mu}^{\dagger} \subset F_{\lambda+\mu}^{\dagger} .
$$

Proof. Using formula (2.6) for any $x \in \mathfrak{g}, r \in \mathbb{Z}_{+}$we have:

$$
x t^{k}\left(\left(F_{\lambda}^{\dagger}\right)_{(n)} F_{\mu}^{\dagger}\right) \subset \sum_{i \in \mathbb{Z}}\left(F_{\lambda}^{\dagger}\right)_{(n+i)} F_{\mu}^{\dagger} .
$$

In other words $\sum_{i \in \mathbb{Z}}\left(F_{\lambda}^{\dagger}\right)_{(n+i)} F_{\mu}^{\dagger}$ is $\mathfrak{g}[t]$-submodule. However for any scalar current $h \in \mathfrak{g}$ over Cartan subalgebra:

$$
h\left(A_{(n)} B\right)=(h \cdot A)_{(n)} B+A_{(n)}(h \cdot B) .
$$

Therefore the set of weights of $\sum_{i \in \mathbb{Z}}\left(F_{\lambda}^{\dagger}\right)_{(n+i)} F_{\mu}^{\dagger}$ is contained in the sum of the sets of weights of $F_{\lambda}^{\dagger}$ and $F_{\mu}^{\dagger}$. In particular, all weights of this $\mathfrak{g}[t]$ submodule are less than or equal to $\lambda$. Thus this submodule is contained in $F_{\lambda+\mu}^{\dagger}$.

Lemma 4.4. For all $\lambda \in P_{+}$one has $G_{\lambda} \subset F_{\lambda}^{\dagger}$.

Proof. We prove the claim by induction with respect to the partial order (1.1). The $\lambda=0$ case is obvious. Now assume our lemma is proved for all weights less than or equal to $\lambda$. In order to prove the inclusion $G_{\lambda} \subset F_{\lambda}^{\dagger}$ it suffices to show that all the weights of the $\mathrm{U}(\mathfrak{g}[t])$-span of $G_{\lambda}$ are less than or equal to $\lambda$. Recall definition (4.1). We first note that all the weights of $G_{\lambda}$ are less than or equal to $\lambda$ (see Proposition 1.8). In fact, let $B \in \widetilde{W}_{\omega_{i}^{*}}^{*}$, $C \in G_{\lambda-\omega_{i}}$ (we assume $\lambda-\omega_{i} \in P_{+}$). Then for any $h \in \mathfrak{h}$ and $k \in \frac{1}{N} \mathbb{Z}$ one has

$$
h\left(B_{(k)} C\right)=B_{(k)}(h . C)+(h . B)_{(k)} C,
$$

which gives the statement by the induction assumption. 
Now assume (by induction) that for all weights $\mu<\lambda$ the $\mathrm{U}(\mathfrak{g}[t])$-span of $G_{\mu}$ is contained in $G_{\mu}+F_{<\mu}$. Then for $B \in \widetilde{W}_{\omega_{i}^{*}}^{*}, C \in G_{\lambda-\omega_{i}}$ and $x \in \mathfrak{g}$, $m \geq 0$ one has (see Corollary 2.6)

$$
x t^{m}\left(B_{(k)} C\right)=B_{(k)}\left(x t^{m} \cdot C\right)+\sum_{j=0}^{m}\left(\begin{array}{c}
m \\
j
\end{array}\right)\left(x t^{j} \cdot B\right)_{(m+k-j)} C,
$$

which belongs to $G_{\lambda}+F_{<\lambda}^{\dagger}$. Therefore $G_{\lambda} \subset F_{\lambda}^{\dagger}$ by Proposition 1.8. This completes the proof of Lemma

4.2. Multiplication. Let $\lambda=\sum_{k=1}^{s} \omega_{j_{k}} \in P_{+}$. Let $\tilde{A}^{k} \in \widetilde{W}_{\omega_{j_{k}}^{*}}^{*}, k=1, \ldots, s$ be a collection of elements in the lifts of the local Weyl modules. For $i, j=$ $1, \ldots, r$ let $m_{i, j}=-\left(\omega_{i}^{*}, \omega_{j}^{*}\right)$. We consider the expression

$$
\mathcal{M}\left(\tilde{A}^{1}, \ldots, \tilde{A}^{s}\right)=\prod_{1 \leq k<l \leq s} i_{z_{k}, z_{l}}\left(z_{k}-z_{l}\right)^{m_{i_{k}, i_{l}}} \prod_{k=1}^{s} Y\left(\tilde{A}^{k}, z_{k}\right)|0\rangle
$$

(see e.g. [DL, Chapter 7, where the matrix coefficients of this expression are considered). Let

$$
\mathcal{M}\left(\tilde{A}^{1}, \ldots, \tilde{A}^{s}\right)=\sum_{m_{1}, \ldots, m_{s}} \mathcal{M}\left(\tilde{A}^{1}, \ldots, \tilde{A}^{s}\right)_{m_{1}, \ldots, m_{s}} z_{1}^{m_{1}} \ldots z_{s}^{m_{s}} .
$$

Lemma 4.5. $\mathcal{M}\left(\tilde{A}^{1}, \ldots, \tilde{A}^{s}\right)_{m_{1}, \ldots, m_{s}} \in G_{\lambda}$ for all $m_{1}, \ldots, m_{s}$.

Proof. Follows from the definition of $G_{\lambda}$.

Lemma 4.6. $\mathcal{M}\left(e^{\omega_{j_{1}}^{*}}, \ldots, e^{\omega_{j_{s}}^{*}}\right)$ is invariant under the permutation of indices $j_{k}$.

Proof. Corollary 2.7 says that

$$
\begin{aligned}
& i_{z_{a}, z_{a+1}}\left(z_{a}-z_{a+1}\right)^{m_{j_{a}, j_{a+1}}} Y\left(e^{\omega_{j_{a}}^{*}}, z_{a}\right) Y\left(e^{\omega_{j_{a+1}}^{*}}, z_{a+1}\right)= \\
& \quad \nu\left(p\left(\omega_{j_{a}}^{*}\right), p\left(\omega_{j_{a+1}}^{*}\right)\right) i_{z_{a+1}, z_{a}}\left(z_{a}-z_{a+1}\right)^{m_{j_{a}, j_{a+1}}} Y\left(e^{\omega_{j_{a+1}}^{*}}, z_{a+1}\right) Y\left(e^{\omega_{j a}^{*}}, z_{a}\right) .
\end{aligned}
$$

Now it suffices to note that

$$
\nu\left(p\left(\omega_{j_{a}}^{*}\right), p\left(\omega_{j_{a+1}}^{*}\right)\right) i_{z_{a+1}, z_{a}}\left(z_{a}-z_{a+1}\right)^{m_{j_{a}, j_{a+1}}}=i_{z_{a+1}, z_{a}}\left(z_{a+1}-z_{a}\right)^{m_{j_{a}, j_{a+1}}} .
$$

Recall the definition 4.3 of the elements $\mathcal{M}\left(\tilde{A}^{1}, \ldots, \tilde{A}^{s}\right)_{m_{1}, \ldots, m_{s}}$.

Lemma 4.7. $\mathcal{M}\left(e^{\omega_{j_{1}}^{*}}, \ldots, e^{\omega_{j_{s}}^{*}}\right) \in \mathbb{L}\left[\left[z_{1}, \ldots, z_{s}\right]\right]$ and

$$
\mathcal{M}\left(e^{\omega_{j_{1}}^{*}}, \ldots, e^{\omega_{j_{s}}^{*}}\right)_{0, \ldots, 0}= \pm e^{\lambda} .
$$

Proof. To prove the first claim, we start with $z_{s}$. Note that $Y\left(e^{\omega_{j_{s}}^{*}}, z_{s}\right)|0\rangle \in$ $\mathbb{L}\left[\left[z_{s}\right]\right]$ and

$$
\prod_{1 \leq k<l \leq s} i_{z_{k}, z_{l}}\left(z_{k}-z_{l}\right)^{m_{j_{k}, j_{l}}}
$$


is a Taylor series in $z_{s}$. Now Lemma 4.6 implies the first claim. The equality (4.4) can be derived from the explicit formula (2.3) for the action of vertex operators $Y\left(e^{\omega_{j}^{*}}, z\right)$.

4.3. The map. Recall the identification $\mathbb{W}_{\omega_{i}^{*}}^{*} \simeq W_{\omega_{i}^{*}}^{*}\left[t^{-1}\right]$. For an element $A \in W_{\omega_{i}^{*}}^{*}$ let $\tilde{A} \in \widetilde{W}_{\omega_{i}^{*}}^{*}$ be its lift in $F_{\omega_{i}}^{\dagger}$. For a dominant integral weight $\lambda=\sum_{k=1}^{s} \omega_{j_{k}}$ we define a map

$$
\begin{gathered}
\varphi: \mathbb{W}_{\omega_{j_{1}}^{*}}^{*} \otimes \ldots \otimes \mathbb{W}_{\omega_{j_{s}}^{*}}^{*} \rightarrow G_{\lambda} /\left(G_{\lambda} \cap F_{<\lambda}^{\dagger}\right), \\
\tilde{A}^{1} t^{-m_{1}} \otimes \ldots \otimes \tilde{A}^{s} t^{-m_{s}} \mapsto\left[\mathcal{M}\left(\tilde{A}^{1}, \ldots, \tilde{A}^{s}\right)_{m_{1}, \ldots, m_{s}}\right]
\end{gathered}
$$

(the square brackets denote the class of an element of $G_{\lambda}$ in $G_{\lambda} /\left(G_{\lambda} \cap F_{<\lambda}^{\dagger}\right)$ ).

Lemma 4.8. The map $\varphi$ does not depend on the lifts $\widetilde{W}_{\omega_{i}^{*}}^{*} \subset F_{\omega_{i}}^{\dagger}$.

Proof. For $A^{i} \in W_{\omega_{i}^{*}}^{*}$ let us choose two lifts $\tilde{A}^{i}$ and $\tilde{\tilde{A}}^{i}$. Then $\tilde{A}^{i}-\tilde{\tilde{A}}^{i} \in F_{<\omega_{i}}^{\dagger}$ and the claim holds because of Proposition 4.3 .

Lemma 4.9. Let us fix $A^{l} \in W_{\omega_{j_{l}}^{*}}^{*}, l=1, \ldots, s$ and $x t^{m} \in \mathfrak{g}[t] \quad(x \in \mathfrak{g}$, $m \geq 0)$. Then

$$
\begin{aligned}
& x t^{m} \cdot \mathcal{M}\left(\tilde{A}^{1}, \ldots, \tilde{A}^{s}\right)_{m_{1}, \ldots, m_{s}}= \\
& \sum_{k=1}^{s} \sum_{p=0}^{m}\left(\begin{array}{c}
m \\
p
\end{array}\right) \mathcal{M}\left(\tilde{A}^{1}, \ldots, x t^{p} . \tilde{A}^{k}, \ldots, \tilde{A}^{s}\right)_{m_{1}, \ldots, m_{k}-m, \ldots, m_{s} .} .
\end{aligned}
$$

Proof. Let us compute the left hand side. Since $x t^{m}|0\rangle=0$, one has

$$
\begin{aligned}
& x t^{m} \cdot \prod_{1 \leq k<l \leq s} i_{z_{k}, z_{l}}\left(z_{k}-z_{l}\right)^{m_{j_{k}, j_{l}}} \prod_{k=1}^{s} Y\left(A^{k}, z_{k}\right)|0\rangle \\
= & \prod_{1 \leq k<l \leq s} i_{z_{k}, z_{l}}\left(z_{k}-z_{l}\right)^{m_{j_{k}, j_{l}}} \sum_{l=1}^{s} Y\left(A^{1}, z_{1}\right) \ldots\left[x t^{m}, Y\left(A^{l}, z_{l}\right)\right] \ldots Y\left(A^{s}, z_{s}\right)|0\rangle .
\end{aligned}
$$

Now by (2.6) one has

$$
\left[x t^{m}, Y\left(A^{l}, z_{l}\right)\right]=\sum_{p=0}^{m}\left(\begin{array}{c}
m \\
p
\end{array}\right) Y\left(x t^{p} \cdot A^{l}, z_{l}\right) z_{l}^{m-p} .
$$

Proposition 4.10. Fix a collection of integers $m_{1}, \ldots, m_{s}$ and assume that $m_{k}<0$ for some $1 \leq k \leq s$. Then

$$
\mathcal{M}\left(\tilde{A}^{1}, \ldots, \tilde{A}^{s}\right)_{m_{1}, \ldots, m_{s}} \in F_{<\lambda}^{\dagger} .
$$

Proof. Thanks to Proposition 1.8 it suffices to show that the weights of the $\mathrm{U}(\mathfrak{g}[t])$-span of $\mathcal{M}\left(\tilde{A}^{1}, \ldots, \tilde{A}^{s}\right)_{m_{1}, \ldots, m_{s}}$ are smaller than $\lambda$. Note that this 
span lies in the linear span of elements $\mathcal{M}\left(\tilde{B}^{1}, \ldots, \tilde{B}^{s}\right)_{a_{1}, \ldots, a_{s}}, B^{l} \in \widetilde{W}_{\omega_{j_{l}}^{*}}^{*}$ and the weights of these elements are smaller than to equal $\leq \lambda$. We note that if the weight of $\mathcal{M}\left(\tilde{B}^{1}, \ldots, \tilde{B}^{s}\right)_{m_{1}, \ldots, m_{s}}, B^{l} \in W_{\omega_{j_{l}}^{*}}^{*}$ is equal to $\lambda$ then the weight of $B^{l}$ is $\omega_{j_{l}}^{*}$. Hence all the weight $\lambda$ vectors in the $\mathrm{U}(\mathfrak{g}[t])$-span of the elements $\mathcal{M}\left(\tilde{A}^{1}, \ldots, \tilde{A}^{s}\right)_{m_{1}, \ldots, m_{s}}$ are of the form

$$
\mathcal{M}\left(e^{\omega_{j_{1}}^{*}}, \ldots, e^{\omega_{j_{s}}^{*}}\right)_{a_{1}, \ldots, a_{s}} .
$$

However, Lemma 4.9 implies that $a_{k}<0$ (since $m \geq 0$ and $m_{k}<0$ ). Now Lemma 4.6 and Lemma 4.7 imply our Proposition.

Theorem 4.11. The map $\varphi$ is a homomorphism of $\mathfrak{g}[t]$ modules.

Proof. Lemma 4.9 and Proposition 4.10 imply

$$
\begin{aligned}
& x t^{m} \cdot\left[\mathcal{M}\left(\tilde{A}^{1}, \ldots, \tilde{A}^{s}\right)_{m_{1}, \ldots, m_{s}}\right]= \\
& \sum_{k=1}^{s} \sum_{p=0}^{m}\left(\begin{array}{c}
m \\
p
\end{array}\right)\left[\mathcal{M}\left(\tilde{A}^{1}, \ldots, x t^{p} . \tilde{A}^{k}, \ldots, \tilde{A}^{s}\right)_{m_{1}, \ldots, m_{k}-m, \ldots, m_{s}}\right],
\end{aligned}
$$

where the terms in the right hand side with $m_{k}<m$ vanish. Now we derive the theorem from formula (1.5) and Proposition 1.4.

Corollary 4.12. The image of $\varphi$ surjects onto $\mathbb{W}_{\lambda^{*}}^{*}$.

Proof. We have a surjection $S$ of $\mathfrak{g}[t]$ modules $\left(\lambda^{*}=\sum_{k=1}^{s} \omega_{j_{k}}^{*}\right)$ :

$$
S: \mathbb{W}_{\omega_{j_{1}}^{*}}^{*} \otimes \ldots \otimes \mathbb{W}_{\omega_{j_{s}}^{*}}^{*} \mapsto \mathbb{W}_{\lambda^{*}}^{*}
$$

We claim that $S(\operatorname{ker} \varphi)=0$. Indeed, assume there exists $y \in \operatorname{ker} \varphi$ such that $S(y) \neq 0$. Since $\mathbb{W}_{\lambda^{*}}^{*}$ is cocyclic $\mathfrak{g}[t]$ module, there exists $\tau \in \mathrm{U}(\mathfrak{g}[t])$ such that $\tau . S(y)$ is the cocylcic vector, which is mapped to a non-zero vector thanks to (4.4), giving a contradiction. We thus obtain an induced surjective map

$$
\mathbb{W}_{\omega_{j_{1}}^{*}}^{*} \otimes \ldots \otimes \mathbb{W}_{\omega_{j_{s}}^{*}}^{*} / \operatorname{ker} \varphi \rightarrow \mathbb{W}_{\lambda^{*}}^{*}
$$

Corollary 4.13. $G_{\lambda}=F_{\lambda}^{\dagger}$ for $\lambda \in P_{+}$.

Proof. Since the image of $\varphi$ is contained in $G_{\lambda} /\left(G_{\lambda} \cap F_{<\lambda}^{\dagger}\right)$, Corollary 4.12 implies the surjection $G_{\lambda} /\left(G_{\lambda} \cap F_{<\lambda}^{\dagger}\right) \rightarrow \mathbb{W}_{\lambda^{*}}^{*}$. However, $G_{\lambda}$ is contained in $F_{\lambda}^{\dagger}$ and $F_{\lambda}^{\dagger} / F_{<\lambda}^{\dagger} \simeq \mathbb{W}_{\lambda^{*}}^{*}$.

We thus obtain the following theorem. Recall the canonical embedding $W_{\lambda}^{*} \subset \mathbb{W}_{\lambda}^{*}$.

Theorem 4.14. The homogeneous coordinate ring $\mathbb{W}^{*}=\bigoplus_{\lambda \in P_{+}} \mathbb{W}_{\lambda}^{*}$ has a structure of $P / Q$-graded vertex operator algebra generated by the fields $Y(A, z), A \in W_{\omega_{i}}^{*}, i=1, \ldots, r$. 
Proof. We know (see Corollary 4.13) that $\operatorname{gr} G_{\bullet}=\operatorname{gr} F_{\bullet}^{\dagger} \simeq \mathbb{W}^{*}$. Since $\left(F_{\lambda}^{\dagger}\right)_{(n)} F_{\mu}^{\dagger} \subset F_{\lambda+\mu}^{\dagger}$, the associated graded space gr $F_{\bullet}^{\dagger}$ carries the $P / Q$-graded vertex operator algebra structure. Finally, the algebra $\operatorname{gr} G_{\bullet}$ is generated by the fields $Y(A, z), A \in W_{\omega_{i}}^{*}, i=1, \ldots, r$ by definition. This completes the proof.

Remark 4.15. Assume that $\mathfrak{g}$ is of type $E_{8}$. Then $\Gamma$ is a trivial group so we deal with the usual vertex algebra. Let $\lambda=\sum_{i=1}^{s} \omega_{j_{i}}$. For any $\tilde{A}^{l} \in \widetilde{W}_{\omega_{j_{l}}}$ we have $\tilde{A}_{(n)}^{l} F_{\lambda}^{\dagger} \subset F_{<\lambda+\omega_{i}}^{\dagger}, n \geq 0$. Therefore for $B=\tilde{A}_{\left(-1-m_{1}\right)}^{1} \ldots \tilde{A}_{\left(-1-m_{s}\right)}^{s}|0\rangle$ one has $B_{(n)} F_{\mu}^{\dagger} \subset F_{<\mu+\lambda}^{\dagger}, n \geq 0$ (this is true only for $\Gamma=\{0\}$ ). Thus in the described above vertex algebra on the space $\bigoplus_{\lambda \in P_{+}} \mathbb{W}_{\lambda}^{*}$ we have:

$$
B_{(n)} C=0, n \geq 0, B, C \in \mathbb{L} .
$$

Therefore this vertex algebra is holomorphic (aka commutative). Hence the bilinear operation $(B, C) \mapsto B_{(-1)} C$ is a commutative and associative, inducing another structure of commutative and associative algebra on the space $\bigoplus_{\lambda \in P_{+}} \mathbb{W}_{\lambda}^{*}$. However in this algebra all the elements $P \otimes e^{\lambda}$ are nilpotent, if $\lambda \neq 0$.

\section{Semi-Infinite Plǘcker Relations via vertex algebras}

In this section we use the vertex operator realization of the homogeneous coordinate ring of the semi-infinite flag variety in order to derive semi-infinite Plücker relations.

Recall $m_{i_{1}, i_{2}}=-\left(\omega_{i_{1}}^{*}, \omega_{i_{2}}^{*}\right)$ and let us fix two vectors $A \in W_{\omega_{i_{1}}^{*}}^{*}, B \in W_{\omega_{i_{2}}^{*}}^{*}$.

Lemma 5.1. The following equality holds in the $P / Q$-graded VOA $\mathbb{W}^{*}$ :

$$
\begin{aligned}
& i_{z, w}(z-w)^{m_{i_{1}, i_{2}} Y(A, z) Y(B, w)|0\rangle} \\
& \quad=\sum_{p, q \geq 0} z^{p} w^{q} \sum_{j \geq 0}(-1)^{j}\left(\begin{array}{c}
m_{i_{1}, i_{2}} \\
j
\end{array}\right) A_{\left(m_{i_{1}, i_{2}}-1-p-j\right)} B_{(-1-q+j)}|0\rangle .
\end{aligned}
$$

Proof. A direct computation shows that the left hand side of (5.1) considered as operators in $\mathbb{L}$ is given by the right hand side with the only change $p, q \in \mathbb{Z}$ (instead of $p, q \in \mathbb{Z}_{\geq 0}$ ). Now applying Proposition 4.10 we derive our Lemma.

Remark 5.2. Recall that the conformal weight of $e^{\lambda} \in \mathbb{L}$ (the cocylic vector of $\left.\mathbb{W}_{\lambda}^{*}\right)$ is equal to $(\lambda, \lambda) / 2$. If $A=e^{\omega_{i_{1}}^{*}}, B=e^{\omega_{i_{2}}^{*}}$, then $A_{m_{i_{1}, i_{2}-1} B}$ is the cocylic vector if $\mathbb{W}_{\omega_{i_{1}}^{*}+\omega_{i_{2}}^{*}}^{*}$, which is equal to $e^{\omega_{i_{1}}^{*}+\omega_{i_{2}}^{*}}$ up to a sign (this can be seen from the explicit formula (2.3) for vertex operators). If $s \leq 0$, then for any $\tilde{A} \in W_{\omega_{i_{1}}^{*}}^{*}, \tilde{B} \in W_{\omega_{i_{2}}^{*}}^{*}$ vector $\tilde{A}_{\left(m_{i_{1}, i_{2}}-s\right)} \tilde{B}$ belongs to $G_{<\left(\omega_{i_{1}}+\omega_{i_{2}}\right)}$.

We prove the following theorem. 
Proposition 5.3. For any $s>0, r \geq 0$ the vector

$$
\left(A_{\left(m_{i_{1}, i_{2}}-s\right)} B\right)_{(-1-r)}|0\rangle
$$

is equal to the coefficient of $z^{r}$ in

$$
\left.\left(i_{z, w}(z-w)^{m_{i_{1}, i_{2}}} \partial_{z}^{s-1} Y(A, z) Y(B, w)\right)\right|_{z=w}|0\rangle
$$

in the $P / Q$-graded $V O A \mathbb{W}^{*}$.

Proof. We use formula (2.1). Let $c$ be the vacuum vector $|0\rangle, m=0, n=$ $m_{i_{1}, i_{2}}-s, k=-r-1, a=A, b=B$. Then the left hand side sum of (2.1) reduces to a single $j=0$ term, which is equal to $\left(A_{\left(m_{i_{1}, i_{2}}-s\right)} B\right)_{(-1-r)}|0\rangle$. In the right hand side all the terms $B_{(n+k-j)} A_{(m+j)}|0\rangle$ vanish since $m+j \geq 0$. Hence we are left with the equality

$$
\left(A_{\left(m_{i_{1}, i_{2}}-s\right)} B\right)_{(-1-r)}|0\rangle=\sum_{j \geq 0}(-1)^{j}\left(\begin{array}{c}
m_{i_{1}, i_{2}}-s \\
j
\end{array}\right) A_{\left(m_{\left.i_{1}, i_{2}-s-j\right)}\right.} B_{(-1-r+j)}|0\rangle .
$$

Now formula (5.1) implies that the coefficient in front of $z^{k}$ in (5.2) is equal to

$$
\sum_{l \geq 0}(-1)^{l}\left(\begin{array}{c}
m_{i_{1}, i_{2}} \\
l
\end{array}\right) \sum_{\substack{p \geq s-1, q \geq 0 \\
p+q=r+s-1}} p(p-1) \ldots(p-s+2) A_{\left(m_{i_{1}, i_{2}}-1-p-j\right)} B_{(-1-q+l)}|0\rangle
$$

(we note that $p, q$ are nonnegative here thanks to Proposition 4.10 which says that no coefficients in front of negative powers of $z, w$ survive in $\mathbb{W}^{*}$ ). So we are left to show that

$$
\begin{aligned}
& \sum_{j \geq 0}(-1)^{j}\left(\begin{array}{c}
m_{i_{1}, i_{2}}-s \\
j
\end{array}\right) A_{\left(m_{i_{1}, i_{2}}-s-j\right)} B_{(-1-r+j)} \\
= & \sum_{l \geq 0}(-1)^{l}\left(\begin{array}{c}
m_{i_{1}, i_{2}} \\
l
\end{array}\right) \sum_{\substack{p \geq s-1, q \geq 0 \\
p+q=r+s-1}} p(p-1) \ldots(p-s+2) A_{\left(m_{i_{1}, i_{2}}-1-p-j\right)} B_{(-1-q+l)} .
\end{aligned}
$$

Combining the terms on the right, the equality is implied by

$$
(-1)^{j}\left(\begin{array}{c}
m_{i_{1}, i_{2}}-s \\
j
\end{array}\right)=\sum_{l=0}^{j}(-1)^{l}\left(\begin{array}{c}
m_{i_{1}, i_{2}} \\
l
\end{array}\right)(j-l+s-1)(j-l+s-2) \ldots(j-l+1) .
$$

The proof of (5.4) is standard: the generating function over $j$ of the left hand side terms is equal to $(1-x)^{m_{i_{1}, i_{2}}-s}$, while on the right the generating

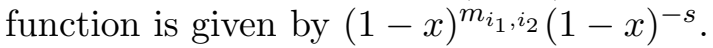

Given an element $A \in W_{\omega_{i}^{*}}^{*}$ we denote by $A(z)=\sum_{r \geq 0} z^{r}\left(A \otimes t^{-r}\right) \in$ $\mathbb{W}_{\omega_{i}^{*}}^{*}[[z]]$ (we use the identification $\mathbb{W}_{\omega_{i}^{*}}^{*} \simeq W_{\omega_{i}^{*}}^{*}\left[t^{-1}\right]$ from Proposition [1.4). Recall the isomorphism of vector spaces $\mathbb{W}^{*} \simeq \operatorname{gr} G_{\bullet}$. We rephrase Theorem 5.3 in terms of multiplication in algebra $\mathbb{W}^{*}$. 
Corollary 5.4. For any $s>0$ and $A \in W_{\omega_{i_{1}}^{*}}^{*}, B \in W_{\omega_{i_{2}}^{*}}^{*}$ one has

$$
\frac{\partial^{s-1} A(z)}{\partial z^{s-1}} B(z)=\sum_{r \geq 0} z^{r}\left(A_{\left(m_{i_{1}, i_{2}}-s\right)} B\right)_{(-1-r)}|0\rangle,
$$

where the multiplication in the left hand side is understood inside $\mathbb{W}^{*}$ and in the right hand side the VOA structure on $\mathbb{W}^{*}$ is used.

Proof. Follows from the multiplication formula (4.2).

We apply Corollary 5.4 to the question of finding relations in the algebra $\mathbb{W}^{*}$ (the so called semi-infinite Plücker relations).

Theorem 5.5. Assume that for some positive $s$ one has

$$
\sum_{l} \tilde{A}_{\left(m_{i_{1}, i_{2}}-s\right)}^{l} \tilde{B}^{l} \in G_{<\left(\omega_{i_{1}}+\omega_{i_{2}}\right)}
$$

for some $A^{l} \in W_{\omega_{i_{1}}^{*}}^{*}, B^{l} \in \tilde{W}_{\omega_{i_{2}}^{*}}^{*}$. Then all the coefficients of the series $\sum_{l}\left(\partial^{s-1} A^{l}(z)\right) B^{l}(z)$ vanish as elements of $\mathbb{W}^{*}$.

Proof. Follows from Corollary 5.4.

Conjecture 5.6. The relations on $\mathbb{W}^{*}$ from Theorem 5.5 are defining.

We prove this conjecture in type $A$ in the section below.

\section{TYPE A}

In this section we consider the case $\mathfrak{g}=\mathfrak{s l}_{r+1}$. The whole picture simplifies a lot because the local Weyl modules $W_{\omega_{i}}$ are irreducible as $\mathfrak{g}$ modules (i.e. $\left.W_{\omega_{i}} \simeq V_{\omega_{i}}\right)$.

Let $\Lambda_{1}, \ldots, \Lambda_{r}$ be the level one integrable weights of $\widehat{\mathfrak{g}}$ such that the restriction of $\Lambda_{i}$ to the Cartan subalgebra of $\mathfrak{g}$ is equal to $\omega_{i}$. Let $v_{\Lambda_{i}} \in L\left(\Lambda_{i}\right)$ be the highest weight vector.

Lemma 6.1. For each $i=1, \ldots, r$ the lift $\widetilde{W}_{\omega_{i}}^{*}$ is unique. One has

$$
\widetilde{W}_{\omega_{i}}^{*}=\mathrm{U}(\mathfrak{g}) v_{\Lambda_{i}} \subset L\left(\Lambda_{i}\right) .
$$

Proof. In type $A$ the inequality $\lambda \leq \omega_{i}$ implies $\lambda=\omega_{i}$ for $\lambda \in P_{+}$.

Corollary 6.2. The space $F_{\omega_{i}}^{\dagger}=G_{\omega_{i}}$ is isomorphic to $\mathbb{W}_{\omega_{i}^{*}}^{*}$ and is explicitly given by

$$
\operatorname{span}\left\{A_{(n)}|0\rangle, A \in W_{\omega_{i}^{*}}^{*}, n \in \mathbb{Z}\right\} .
$$

Let us now explicitly describe the relations for the algebra $\mathbb{W}^{*}$. In [FM2] for any two strictly increasing columns $I$ and $J$ such that length of $I$ is no smaller than the length of $J$ we defined the number $k(I, J)$. The value $k(I, J)$ measures how far the two-column Young tableaux $Y(I, J)$ corresponding to the pair $I, J$ is from being semi-standard. In particular, $k(I, J)=0$ if and 
only if the corresponding tableaux is semi-standard. Let us recall the formal definition.

Let $I, J \subset\{1, \ldots, r+1\}, I=\left(i_{1}<\cdots<i_{l(I)}\right), J=\left(j_{1}<\cdots<j_{l(J)}\right)$. We assume that either $l(I)>l(J)$, or $l(I)=l(J)$ and there exists $1 \leq s \leq l(I)$ such that

$$
i_{l(I)}=j_{l(I)}, \ldots, i_{s+1}=j_{s+1}, i_{s}<j_{s}
$$

We construct a set $P=\left(p_{1}, \ldots, p_{M}\right) \subset I \sqcup J$ step by step decreasing the index. We start with fixing $p_{M}=i_{l(I)}$. Now at each step $p_{k}$ is identified with an element $i_{a} \in I$ or $j_{b} \in J$. We proceed via the following rule:

- if $p_{k}=i_{a}$ and $i_{a}>j_{a}$, then $p_{k-1}=j_{a}$; otherwise $p_{k-1}=i_{a-1}$,

- if $p_{k}=j_{a}$ and $i_{a}<j_{a}$, then $p_{k-1}=i_{a}$; otherwise $p_{k-1}=j_{a-1}$.

Definition 6.3. $k(I, J)=|P(I, J)|-l(I)$.

Let $Y(I, J)$ be the two-column Young diagram whose left (longer) column is $I$ and the right column is $J$.

Lemma 6.4. The following properties hold by construction:

- $Y(I, J)$ is semi-standard if and only if $k(I, J)=0$,

- $p_{1}<p_{2}<\cdots<p_{M}$,

- $0 \leq k(I, J) \leq \min (l(J), r+1-l(I))$.

Proof. To prove the first claim we note that $k(I, J)=0$ is equivalent to $P(I, J)=I$, which means that $i_{a} \leq j_{a}$ for all $a$. The second claim immediately follows from the definition. Since all the numbers in $P$ are distinct and belong to $\{1, \ldots, r+1\}$, we conclude $k(I, J)+l(I) \leq r+1$. The inequality $k(I, J) \leq l(J)$ is obvious, since it is equivalent to $|P(I, J)| \leq l(I)+l(J)$.

In what follows we use the standard notation $\omega_{i}=\sum_{a=1}^{i} \varepsilon_{a}$. For a Young tableau $T$ the weight of $T$ is the linear combination $\sum_{a=1}^{r+1} m_{a} \varepsilon_{a}$, where $m_{a}$ is the number of times $a$ shows up in $T$.

Lemma 6.5. Let us fix $i \geq j$. Then for any $0 \leq l \leq \min (j, r+1-i)$ there exists a two column Young tableau $T=(C, D)$ of shape $\omega_{i}+\omega_{j}$ with the left column $C=\left(c_{1}<\cdots<c_{i}\right)$ and right column $D=\left(d_{1}<\cdots<d_{j}\right)$ such that $k(C, D)=l$ and the weight of $T$ is equal to $\omega_{i+l}+\omega_{j-l}$.

Proof. We need to fill $T=(C, D)$ with the numbers $1, \ldots, r+1$ in such a way that each of the numbers $1, \ldots, j-l$ shows up twice and each of the numbers $j-l+1, \ldots, i+l$ shows up once. Here is the rule: we consider the numbers $1, \ldots, i+l$ and start inserting these numbers into $T$ moving from bottom to top in the following way: we first define

$$
c_{i}=i+l, c_{i-1}=i+l-1, \ldots, c_{j}=j+l .
$$

Then we turn right, making a horizontal move, i.e. define $d_{j}=j+l-1$ and then go up, setting $d_{j-1}=j+l-2$. Then we make a horizontal move by $c_{j-1}=j+l-3$ and a vertical step $c_{j-2}=j+l-4$ etc. until we make exactly 
EVGENY FEIGIN AND IEVGEN MAKEDONSKYI

$l$ horizontal moves. Then we get $c_{j-l+1}=j-l+1$ or $d_{j-l+1}=j-l+1$. We finalize the procedure by setting

$$
c_{j-l-s+1}=d_{j-l-s+1}=j-l-s+1, s=0, \ldots, j-l .
$$

Here are examples of the tableaux $T$ from Lemma 6.5 for $r=13, i=10$, $j=6$ with $l=0,1,2,3$ :

$\begin{array}{cccccccc}1 & 1 & 1 & 1 & 1 & 1 & 1 & 1 \\ 2 & 2 & 2 & 2 & 2 & 2 & 2 & 2 \\ 3 & 3 & 3 & 3 & 3 & 3 & 3 & 3 \\ 4 & 4 & 4 & 4 & 4 & 4 & 5 & 4 \\ 5 & 5 & 5 & 5 & 5 & 6 & 6 & 7 \\ 6 & 6 & 7 & 6 & 8 & 7 & 9 & 8 \\ 7 & & 8 & & 9 & 10 & \\ 8 & 9 & 10 & 11 & \\ 9 & 10 & 11 & 12 & \\ 10 & 11 & 12 & & 13\end{array}$

Remark 6.6. The proof of Lemma 6.7 below implies that the tableau $T$ constructed in the proof of Lemma 6.5 is unique with the desired properties.

Lemma 6.7. For $1 \leq j \leq i \leq r$ the q-character of the local Weyl module $W_{\omega_{i}+\omega_{j}}$ is given by

$$
\operatorname{ch} W_{\omega_{i}+\omega_{j}}=\sum_{l=0}^{\min (j, r+1-i)} q^{l} \operatorname{ch~} V_{\omega_{j-l}+\omega_{i+l}},
$$

where $\omega_{0}=\omega_{r+1}=0$.

Proof. Recall that one has an isomorphism of $\mathfrak{g}$-modules $W_{\omega_{i}+\omega_{j}}=V_{\omega_{i}} \otimes V_{\omega_{j}}$. The Littlewood-Richardson rule tells us that

$$
V_{\omega_{i}} \otimes V_{\omega_{j}} \simeq \sum_{l=0}^{\min (j, r+1-i)} V_{\omega_{j-l}+\omega_{i+l}} .
$$

So we only need to show that the summand $V_{\omega_{j-l}+\omega_{i+l}}$ shows up at the level $l$ in $W_{\omega_{i}+\omega_{j}}$. Theorem 3.21 of [FM2] gives the following formula for the character of $W_{\omega_{i}+\omega_{j}}\left(\right.$ here $\left.x_{a}=\exp \left(\varepsilon_{a}\right)\right)$ :

$$
\operatorname{ch} W_{\omega_{i}+\omega_{j}}=\sum_{|I|=i,|J|=j} q^{k(I, J)} \prod_{a=1}^{l(I)} x_{i_{a}} \prod_{b=1}^{l(J)} x_{j_{b}} .
$$

We claim that for $1 \leq l \leq \min (j, r+1-i)$

$$
\operatorname{ch~} V_{\omega_{j-l}+\omega_{i+l}}=\sum_{\substack{|I|=i,|J|=j \\ k(I, J)=l}} \prod_{a=1}^{l(I)} x_{i_{a}} \prod_{b=1}^{l(J)} x_{j_{b}},
$$


(for example, (6.1) for $l=0$ simply means that semi-standard tableaux form a basis for the module $V_{\omega_{i}+\omega_{j}}$ ). In fact, the right hand side of (6.1) is a character of a summand of the tensor product $V_{\omega_{i}} \otimes V_{\omega_{j}}$. Thanks to Lemma 6.5 the right hand side of (6.1) contains a term corresponding to the highest weight vector of $V_{\omega_{i+l}+\omega_{j-l}}$. This implies (6.1).

Recall that the algebra $\mathbb{W}^{*}$ is generated by its fundamental part $\bigoplus_{a=1}^{r} \mathbb{W}_{\omega_{a}}^{*}$. Proposition below claims that the vertex operator approach produces the generating set of relations in type $A$. It is tempting to conjecture that the same result hold in all types. Our argument is based on [FM2], which treats type $A$ only. For other types it is not even known if the ideal of relations is generated by quadratic part.

Proposition 6.8. The relations described in Theorem 5.5 generate the ideal of relations of $\mathbb{W}^{*}$.

Proof. Corollary 3.22 and Proposition 2.9 a), [FM2] state that the ideal of relations of $\mathbb{W}^{*}$ is generated by its quadratic part and the generating set of relations can be described as follows: given a non semi-standard two-column Young tableaux $T=(I, J)$ of shape $\omega_{i}+\omega_{j}$ one produces a relation in the coordinate ring $\bigoplus_{\lambda \in P_{+}} V_{\lambda}^{*}$ of the form $R(I, J)=X_{I} X_{J}+\sum_{I^{\prime}, J^{\prime}} \pm X_{I^{\prime}} X_{J^{\prime}}$ (here the Plücker coordinates $X_{I}$ are considered as elements of $V_{\omega_{l(I)}}^{*}$ ). In other words, $R(I, J)$ belongs to the kernel of the map $V_{\omega_{i}}^{*} \otimes V_{\omega_{j}}^{*} \rightarrow V_{\omega_{i}+\omega_{j}}^{*}$. Now the relation $R(I, J)$ produces $k(I, J)$ series of relations of the form

$$
\frac{\partial_{s} X_{I}(z)}{\partial z^{s}} X_{J}(z)+\sum_{I^{\prime}, J^{\prime}} \pm X_{I^{\prime}}(z) X_{J^{\prime}}(z), s<k(I, J),
$$

where $X_{I}(z)=\sum_{k \geq 0}\left(X_{I} t^{-k}\right) z^{k}$ and $X_{I} t^{-k}$ is considered as an element of $W_{\omega_{l(I)}}^{*}\left[t^{-1}\right] \simeq \mathbb{W}_{\omega_{l(I)}^{*}}^{*}$. Corollary 3.22 and Proposition 2.9 a), [FM2] state that the coefficients of the relations above generate the ideal of relations in $\mathbb{W}^{*}$.

For a positive integer $l$ we consider a map

$$
\varphi_{l}: W_{\omega_{i}}^{*} \otimes W_{\omega_{j}}^{*} \rightarrow \mathbb{W}_{\omega_{i}+\omega_{j}}^{*}, \varphi_{l}(A, B)=\left[A_{\left(m_{i, j}-l\right)} B\right]
$$

where as usual the notation $\left[A_{\left(m_{i, j}-l\right)} B\right]$ is used to denote the class of the element $A_{\left(m_{i, j}-l\right)} B$ in $\operatorname{gr} G_{\omega_{i}+\omega_{j}}$. We are interested in the kernel of $\varphi_{l}$. Let $\mathbb{W}_{\omega_{i}+\omega_{j}}^{*}=\sum_{s \geq 0} \mathbb{W}_{\omega_{i}+\omega_{j}}^{*}[s]$ be the decomposition with respect to the $q$-grading. Then $\varphi_{l}$ is a $\mathfrak{g}$-homomorphism from $V_{\omega_{i}}^{*} \otimes V_{\omega_{j}}^{*}$ to $\mathbb{W}_{\omega_{i}+\omega_{j}}^{*}$ thanks to Corollary 2.6 (recall $W_{\omega_{i}} \simeq V_{\omega_{i}}$ ). Thanks to Lemma 6.7 the decomposition of the $\mathfrak{g}$-module $\mathbb{W}_{\omega_{i}^{*}+\omega_{j}^{*}}^{*}[s]$ into irreducible components does not contain direct summands of the form $V_{\omega_{i+l}+\omega_{j-l}}^{*}$ for $l>s$. We conclude that

$$
\operatorname{ker} \varphi_{l} \supset V_{\omega_{i+l}+\omega_{j-l}}^{*} \oplus V_{\omega_{i+l+1}+\omega_{j-l-1}}^{*} \oplus \cdots \oplus V_{\omega_{i+\min (j, r+1-i)}^{*}+\omega_{j-\min (j, r+1-i)}} .
$$


According to Theorem 5.5 for each $l \leq \min (j, r+1-i)$ we obtain the relations of the form

$$
\sum_{m}\left(\partial_{z}^{l-1} A^{m}(z)\right) B^{m}(z), s \leq l
$$

for each element $\sum_{m} A^{m} \otimes B^{m} \in V_{\omega_{i+l}+\omega_{j-l}}^{*} \subset V_{\omega_{i}}^{*} \otimes V_{\omega_{j}}^{*}$.

We claim that all the relations (6.2) can be obtained in this way. Indeed, let us consider relation (6.2) attached to the two column tableau from Lemma 6.5. Then this relation is a highest weight vector of $V_{\omega_{i+l}+\omega_{j-l}}^{*} \subset$ $V_{\omega_{i}}^{*} \otimes V_{\omega_{j}}^{*}$ and thus is contained in (6.4). Now we use the $\mathfrak{g}$-invariance of the relations (6.2) and (6.4).

\section{ACKNOWLEDGMENTS}

The work on Sections 1,2,3 was partially supported by the Russian Academic Excellence Project '5-100'. The work on Sections 4,5,6 was partially supported by the grant RSF-DFG 16-41-01013.

\section{REFERENCES}

[A] T. Arakawa, A remark on the $C_{2}$ cofiniteness condition on vertex algebras, Math.Z., 270(1-2):559-575, 2012.

[BF1] A.Braverman, M.Finkelberg, Weyl modules and $q$-Whittaker functions, Mathematische Annalen, vol. 359 (1), 2014, pp 45-59.

[BF2] A.Braverman, M.Finkelberg, Semi-infinite Schubert varieties and quantum Ktheory of flag manifolds, J. Amer. Math. Soc. 27(2014), no. 4, 1147-1168.

[BG] A. Braverman, D. Gaitsgory, Geometric Eisenstein series, Invent. Math. 150 (2002), 287-384.

[BK] B. Bakalov, V.G. Kac, Generalized vertex algebras, in Lie theory and its applications to physics VI, eds.V.K. Dobrev et al, Heron Press, Sofia, 2006.

[C] R. Carter, Lie Algebras of Finite and Affine Type, Cambridge University Press, 2005.

[CFK] V. Chari, G. Fourier, and T. Khandai, A categorical approach to Weyl modules, Transform. Groups, 15(3):517-549, 2010.

[CI] V.Chari, B.Ion, BGG reciprocity for current algebras, Compositio Mathematica 151 (2015), pp. 1265-1287.

[CL] V. Chari, S. Loktev, Weyl, Demazure and fusion modules for the current algebra of $\mathfrak{s l}_{r+1}$, Adv. Math. 207 (2006), 928-960.

[CP] V. Chari and A. Pressley, Weyl modules for classical and quantum affine algebras, Represent. Theory, 5:191-223 (electronic), 2001.

[Ch] I. Cherednik, Double affine Hecke algebras, London Mathematical Society Lecture Note Series, 319, Cambridge University Press, Cambridge, 2006.

[ChF] I. Cherednik, B. Feigin, Rogers-Ramanujan type identities and Nil-DAHA, Advances in Mathematics 248 (2013), 1050- 1088.

[ChK] I. Cherednik, S. Kato, Nonsymmetric Rogers-Ramanujan sums and thick Demazure modules, arXiv:1802.03819

[DL] C. Dong, J. Lepowsky, Generalized vertex algebras and relative vertex operators, Birkhauser, 1993.

[FB] E. Frenkel, D. Ben-Zvi, Vertex Algebras and Algebraic Curves, 2nd Edition, Mathematical Survey and Monographs 88, A.M.S., 2004.

[FM1] E. Feigin, I. Makedonskyi, Generalized Weyl modules, alcove paths and Macdonald polynomials, Selecta Math.(N.S.) 23 (2017), 2863-2897. 
[FM2] E. Feigin, I. Makedonskyi, Semi-infinite Plücker relations and Weyl modules, arXiv:1709.05674.

[FMO] E. Feigin, I. Makedonskyi, D. Orr, Generalized Weyl modules and nonsymmetric $q$-Whittaker functions, arXiv:1605.01560, to appear in Advances in Mathematics.

[FKM] E. Feigin, S. Kato, I. Makedonskyi, Representation theoretic realization of nonsymmetric Macdonald polynomials at infinity, arXiv:1703.04108.

[FK] I.B. Frenkel, V.G.Kac, Basic representations of affine Lie algebras and dual resonance models, Invent. Math., 62(1):23-66, 1980/81.

[FL] G.Fourier, P.Littelmann, Weyl modules, Demazure modules, KR-modules, crystals, fusion products and limit constructions, Advances in Mathematics 211 (2007), no. 2, 566-593.

[FLM] I.B. Frenkel, J. Lepowsky, J. Meurman, Vertex operator algebras and the Monster, Academic Press, 1988.

[FMi] M.Finkelberg, I.Mirković, Semi-infinite flags I. Case of global curve $\mathbb{P}^{1}$. In Differential topology, infinite-dimensional Lie algebras, and applications, volume 194 of Amer. Math. Soc. Transl. Ser. 2, pages 81-112. Amer. Math. Soc., Providence, RI, 1999.

[I] B. Ion, Nonsymmetric Macdonald polynomials and Demazure characters, Duke Mathematical Journal 116:2 (2003), 299-318.

[Kac1] V. Kac, Infinite dimensional Lie algebras, 3rd ed., Cambridge University Press, Cambridge, 1990.

[Kac2] V. Kac, Vertex Algebras for Beginners, University Lecture Series Vol.10, American Mathematical Society, 1996.

[Kat] S. Kato, Demazure character formula for semi-infinite flag varieties, Math. Ann. (2018). https://doi.org/10.1007/s00208-018-1652-5

[KL] S. Kato, S. Loktev, with an appendix by Ryosuke Kodera, A Weyl Module Stratification of Integrable Representations, arXiv:1712.03508.

[KNS] S. Kato, S. Naito, D. Sagaki, Equivariant K-theory of semi-infinite flag manifolds and Pieri-Chevalley formula, arXiv:1702.02408.

[Kum] S.Kumar, Kac-Moody groups, their flag varieties and representation theory, Progress in Mathematics, 204. Birkhäuser Boston, Inc., Boston, MA, 2002.

[Li1] H. Li, Vertex algebras and vertex Poisson algebras, Commun. Contemp. Math., Vol. 6, No. 1, pp. 61-110, 2004.

[Li2] H. Li, Abelianizing vertex algebras, Comm. Math. Phys., Vol. 259, No. 2, pp.391-411, 2005.

[R] U. Ray, Automorphic forms and Lie superalgebras, 2006, Springer.

[S] N.R. Scheithauer, The fake monster superalgebra, Adv. in Math., Vol. 151, no. 2 (2000), 226-269. 
Evgeny FEIGin:

Department of Mathematics,

National Research University Higher School of Economics,

Usacheva str. 6, 119048, Moscow, Russia,

and

Skolkovo Institute of Science and Technology, Skolkovo Innovation Center, Building 3, Moscow 143026, Russia

E-mail address: evgfeig@gmail.com

IEVGEN MAKEDONSKYI:

Department of Mathematics, Kyoto University, Oiwake, Kita-Shirakawa, Sakyo КYOTO 606-8502, JAPAN

and

DEPARTMENT OF MATHEMATICS,

National Research University Higher School of Economics,

Usacheva str. 6, 119048, Moscow, Russia

E-mail address: makedonskii_e@mail.ru 